Office of Naval Research

Contract N00014-67-A-0298-0006 NR-372-012

NATIONAL AERONAUTICS AND SPACE ADMINISTRATION

Grant NGR 22.007.068

\title{
A DISCRETE-TIME DIFFERENTIAL DYNAMIC PROGRAMMING ALGORITHM WITH APPLICATION TO OPTIMAL ORBIT TRANSFER
}

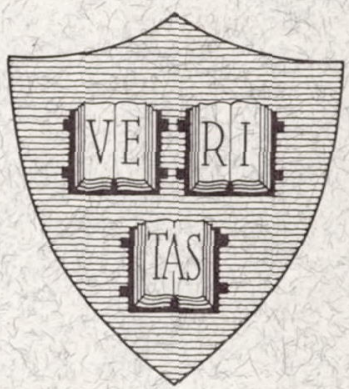

By

Stanley B. Gershwin \& David H. Jacobson

August 1968

Technical Report No. 566

This document has been approved for public

release and sale; its distribution is unlimited.

Division of Engineering and Applied Physics

Harvard University - Cambridge, Massachusetts 
Office of Naval Research

Contract N00014-67-A-0298-0006

NR - $372-012$

National Aeronautics and Space Administration

Grant NGR 22-007-068

A DISCRETE-TIME DIFFERENTIAL DYNAMIC PROGRAMMING

A LGOR ITHM WITH APPLICATION TO OPTIMAL ORBIT TRANSFER

By

Stanley B. Gershwin and David H. Jacobson

Technical R eport No. 566

This document has been approved for public release and sale; its distribution is unlimited.

The research reported in this document was made possible through support extended the Division of Engineering and Applied Physics, Harvard University by the U. S. Army Research Office, the U. S. Air Force Office of 'Scïentific. Research and the U.. S. Office of Naval Research under the Joint Services Electronics Program by Contracts N00014-67-A-0298-0006,0005, and 0008 and by the National Aeronautics and Space Administrationiunder Grant NGR 22-007-068.

Division of Engineering and Applied Physics

Harvard University Cambridge, Massachusetts 


\section{Acknowledgements}

The authors wish to thank Professor A. E. Bryson, Jr. for his valuable suggestions, and Dr.R.G. Tobey of IBM for the use of the FORMAC system, which was helpful in manipulation of the rather complicated algebraic expressions that appear in this paper.

S. B. Gershwin wishes to thank D. H. Jacobson for his patience and accessability during the preparation of this report. 
Page Intentionally Left Blank 


\title{
A DISCRETE-TIME DIFFERENTIAL DYNAMIC PROGRAMMING ALGORITHM WITH APPLICATION TO OPTIMAL ORBIT TRANSFER
}

By

Stanley B. Gershwin and David H. Jacobson

Division of Engineering and Applied Physics

Harvard University Cambridge, Massachusetts

\begin{abstract}
Recently, the notion of Differential Dynamic Programming has been used to obtain new second-order algorithms for solving non-linear optimal control problems. (Unlike conventional Dynamic Programming, the Principle of Pptimality is applied in the neighborhood of a nominal, non-optimal, trajectory.) A novel feature of these algorithms is that they permit strong variations in the system trajectory.

In this paper, Differential Dynamic Programming is used to develop a second-order algorithm for solving discrete-time dynamic optimization problems with terminal constraints. This algorithm also utilizes strong variations and, as a result, has certain advantages over existing discrete-time methods.

A non-linear computed example is presented, and comparisons are made with the results of othex researchers who have solved this problem.

The experience gained during the computation has suggested some extensions to an earlier, previously published Differential Dynamic Programming algorithm for continuous time problems. These extensions, and their implications are discussed.
\end{abstract}


Page Intentionally Left Blank 
Notation

Vectors are columns; the scalar product of $a$ and $b$, where

$$
a=\left[\begin{array}{c}
a_{1} \\
\vdots \\
a_{n}
\end{array}\right] \quad b=\left[\begin{array}{c}
b_{1} \\
\vdots \\
b_{n}
\end{array}\right]
$$

is $a^{T_{b}}$ or $b^{T} a$ and is equal to $\sum_{i=1}^{n} a_{i} b_{i}$. The derivative of a scalar by a vector is a row, and is written:

$$
V_{x}=\frac{\partial V}{\partial x}=\left[\frac{\partial V}{\partial x_{1}}, \ldots, \frac{\partial V}{\partial x_{n}}\right]
$$

The second derivative of a scalar by vectors is a matrix:

$$
\mathrm{v}_{\mathrm{xk}}=\frac{\partial^{2} \mathrm{~V}}{\partial \mathrm{x} \partial \mathrm{k}}=\left[\begin{array}{ccc}
\frac{\partial^{2} \mathrm{~V}}{\partial \mathrm{x}_{1} \partial \mathrm{k}_{1}} & \cdots & \frac{\partial^{2} \mathrm{~V}}{\partial \mathrm{x}_{1} \partial \mathrm{k}_{\mathrm{m}}} \\
\vdots & & \vdots \\
\frac{\partial^{2} \mathrm{~V}}{\partial \mathrm{x}_{\mathrm{n}} \partial \mathrm{k}_{1}} & \cdots & \frac{\partial^{2} \mathrm{~V}}{\partial \mathrm{x}_{\mathrm{n}} \partial \mathrm{k}_{\mathrm{m}}}
\end{array}\right]
$$

where $\mathbf{x}$ is an $n$-vector and $k$ is an $m$-vector.

Thus a second-order Taylor expansion will be written:

$$
\begin{aligned}
\mathrm{V}(\mathrm{x}+\delta \mathrm{x}, \mathrm{k}+\delta \mathrm{k})=\mathrm{V}(\mathrm{x}, \mathrm{k}) & +\mathrm{V}_{\mathrm{x}} \delta \mathrm{x}+\mathrm{v}_{\mathrm{k}} \delta \mathrm{k}+\frac{1}{2} \delta_{\mathrm{x}}^{\mathrm{T}} \mathrm{V}_{\mathrm{xx}} \delta_{\mathrm{x}} \\
+ & \delta_{\mathrm{x}}^{\mathrm{T}} \mathrm{V}_{\mathrm{xk}} \delta \mathrm{k}+\frac{1}{2} \delta_{\mathrm{k}}^{\mathrm{T}} \mathrm{v}_{\mathrm{kk}} \delta \mathrm{k} .
\end{aligned}
$$

$-\mathrm{v}-$ 


\section{Introduction}

Jacobson [1], [2] has derived a second-order algorithm for solving continuous time optimal control problems using Differential Dynamic Programming. This algorithm differs from other second-order or second-variation algorithms, [4], [5], [6], [7], [9], [10], [11], [14] in that it is derived using global variations in control (strong variations in the trajectory).

In this paper a similar algorithm is developed for solving discretetime dynamic optimization problems with terminal constraints. The new algorithm uses the notion of strong variations and hence, as in the case of the continuous time algorithm, has advantages over existing discrete-time algorithms [4], [5], [9], [14]. The algorithm can be used to solve continuous time problems that are approximated by difference equations.

A non-linear numerical example is presented and comparisons are drawn with McReynolds [4], [5] and others [7], [8], who have solved this problem previously, using other methods. The experience gained in the numerical computation has suggested extensions to the continuous algorithms' in [1] and [2]. In particular, the 'step-size adjustment' technique is generalized by the introduction of additional criteria for ensuring that the 'trial new trajectory', at each iteration, is sufficiently close to the current nominal trajectory to guarantee an improvement in cost and/or terminal error. 
II. Derivation of the Discrete Algorithm

II. 1. Statement of the General Problem

The problem to be solved is the following: if $x_{0}, \ldots, x_{N}$ are vector quantities which satisfy

$$
x_{i+1}=f\left(x_{i}, u_{i}, t_{i}\right)
$$

and $x_{0}$ is given, find the vectors $u_{0}, \ldots, u_{N-1}$ to minimize the scalar

$$
\vec{V}=\sum_{i=0}^{N-1} L\left(x_{i}, u_{i}, t_{i}\right)+F\left(x_{N}\right),
$$

where the solution must satisfy the (vector) equality constraint

$$
\theta\left(\mathrm{x}_{\mathrm{N}}\right)=0
$$

$\mathrm{N}$ and $\mathrm{t}_{\mathrm{o}}, \ldots, \mathrm{t}_{\mathrm{N}}$ are known quantities, and a nominal control $\overline{\mathrm{u}}_{\mathrm{o}}, \ldots, \overline{\mathrm{u}}_{\mathrm{N}-1}$ is given.

Defining

$$
\mathrm{V}\left(\mathrm{x}_{\mathrm{o}}, \mathrm{k}, \mathrm{t}_{\mathrm{o}}\right)=\hat{\mathrm{V}}+\mathrm{k}^{\mathrm{T}} \theta \text {, }
$$

the equivalent problem of finding $u_{0}, \ldots, u_{N-1}$ to minimize $V\left(x_{0}, k_{0}, t_{0}\right) t$ and $k$ to satisfy (3) is solved in succeeding sections. A nominal value of $\mathrm{k}, \overline{\mathrm{k}}$, is assumed given.

II. 2. Outline of the Solution

The optimal return function V satisfies Bellman's "Principle of Optimality" [3], which in this case is:

$$
V\left(x_{i}, k, t_{i}\right)=\min _{u_{i}}\left[L\left(x_{i}, u_{i}, t_{i}\right)+V\left(x_{i+1}, k, t_{i+1}\right)\right]
$$

for $i=0, \ldots, N-1$.

Regarded in terms of displacements $\delta_{x_{i}}, \delta_{x_{i+1}}$, and $\delta$ from the nominal trajectory,

$\overline{t \text { It is assumed that a minimum exists. }}$ 


$$
\begin{aligned}
& x_{i}=\bar{x}_{i}+\delta_{x_{i}} \\
& x_{i+1}=\bar{x}_{i+1}+\delta x_{i+1} \\
& k=\bar{k}+\delta k
\end{aligned}
$$

and (5) becomes

$$
\begin{aligned}
v\left(\bar{x}_{i}+\delta x_{i}, \bar{k}+\delta k, t_{i}\right)=\min _{u_{i}}\left[L \left(\bar{x}_{i}\right.\right. & \left.+\delta_{x_{i}}, u_{i}, t_{i}\right) \\
& \left.+V\left(\bar{x}_{i+1}+\delta x_{i+1}, \bar{k}+\delta k, t_{i+1}\right)\right]
\end{aligned}
$$

The algorithm is derived from equation (6) in the following sequence of steps:

1. Expand both sides in Taylor series about $\bar{x}_{i}, \bar{k}$ and $\bar{x}_{i+1}$ in $\delta x_{i}$, $\delta_{\mathrm{k}}$ and $\delta_{\mathrm{x}}+1$

2. Relate $\delta_{x_{i+1}}$ to $\delta_{x_{i}}$.

3. Perform the indicated minimization with respect to $u_{i}$ in two stages.
A. Find $u_{i}^{*}$ which minimizes the right side of $(6)$ with $\delta_{\mathrm{x}_{i}}=0$ and $\delta_{\mathrm{k}}=0$.
B. Expand about $u_{i}^{*}$ in $\delta u_{i}$ with $\delta_{x_{i}}$ and $\delta k$ non-zero, and minimize with respect to $\delta u_{i}$. This will give $\delta u_{i}$ as a function of $\delta x_{i}$ and $\delta k$.

4. Equate coefficients of like powers of $\delta_{x_{i}}$ and $\delta k_{k}$ to obtain difference equations in $\mathrm{V}_{\mathrm{x}}^{\mathrm{i}}, \mathrm{V}_{\mathrm{k}}^{\mathrm{i}}$, etc.

It is assumed that $\delta_{x_{i}}, \delta_{x_{i+1}}$ and $\delta k_{k}$ will be sufficiently small that all Taylor expansions can be terminated at second-order terms. II. 3. Solution

Following the prescription of the previous section, the left side of (6), when expanded in a Taylor series, is, 


$$
\begin{aligned}
V\left(\bar{x}_{i}+\delta_{x_{i}}, \bar{k}+\delta k, t_{i}\right)= & V\left(\bar{x}_{i}, \bar{k}, t_{i}\right)+\frac{\partial}{\partial x} v\left(\bar{x}_{i}, \bar{k}, t_{i}\right) \delta x_{i}+\frac{\partial}{\partial k} v\left(\bar{x}_{i}, \bar{k}, t_{i}\right) \delta_{i} \\
& +\frac{1}{2} \delta x_{i}^{T} \frac{\partial^{2}}{\partial x^{2}} V\left(\bar{x}_{i}, \bar{k}, t_{i}\right) \delta x_{i} \\
& +\delta_{x_{i}}^{T} \frac{\partial^{2}}{\partial x \partial k} V\left(\bar{x}_{i}, \bar{k}, t_{i}\right) \delta k \\
& +\frac{1}{2} \delta k^{T} \frac{\partial^{2}}{\partial k^{2}} v\left(\bar{x}_{i}, \bar{k}, t_{i}\right) \delta k+\ldots
\end{aligned}
$$

The reader should note that $V\left(\bar{x}_{i}, \bar{k}, t_{i}\right)$ is the minimal value of the return function obtainable with initial conditions at $\bar{x}_{i}, t_{i}$, and with $k=\bar{k}$. It is not the same as $\bar{V}\left(\bar{x}_{i}, \bar{k}, t_{i}\right)$, the value of the return function calculated along the nominal trajectory, starting from $t_{i}$.

Symbolically,

$$
V\left(\bar{x}_{i}, \bar{k}, t_{i}\right)=u_{i}, \ldots \min _{N-1}\left[\sum_{j=i}^{N-1} L\left(x_{j}, u_{j}, t_{j}\right)+F\left(x_{N}\right)+\bar{k}^{T} \theta\left(x_{N}\right)\right]
$$

where $x_{i+1}, \ldots, x_{N}$ satisfy $(1)$, and $x_{i}=\bar{x}_{i}$.

However,

$$
\bar{V}\left(\bar{x}_{i}, \bar{k}, t_{i}\right)=\sum_{j=i}^{N-1} L\left(\bar{x}_{j}, \bar{u}_{j}, t_{j}\right)+F\left(\bar{x}_{N}\right)+\bar{k}^{T} \theta\left(\bar{x}_{N}\right)
$$

where $\bar{u}_{i}, \ldots, \bar{u}_{N-1}$ is the nominal control sequence and thus, $\bar{x}_{i}, \ldots, \bar{x}_{N}$ is the nominal trajectory (which satisfies (1) with $\left.u_{j}=\bar{u}_{j}, j=i, \ldots, N-1\right)$.

Acknowledging the difference between $V\left(\bar{x}_{i}, \bar{k}_{,} t_{i}\right)$ and $\bar{V}\left(\bar{x}_{i}, \bar{k}, t_{i}\right)$, define

$$
a\left(\bar{x}_{i}, \bar{k}_{j} ; t_{i}\right)=v\left(\bar{x}_{i}, \bar{k}, t_{i}\right)-\bar{v}\left(\bar{x}_{i}, \bar{k}_{,} t_{i}\right)
$$


To simplify notation, let

$$
\begin{aligned}
& \bar{v}\left(\bar{x}_{i}, \bar{k}, t_{i}\right)=\bar{v}^{i} \\
& V\left(\bar{x}_{i}, \bar{k}, t_{i}\right)=v^{i} \\
& a\left(\bar{x}_{i}, \bar{k}_{,} t_{i}\right)=a^{i} \\
& \frac{\partial}{\partial x} V\left(\bar{x}_{i}, \bar{k}_{1} t_{i}\right)=v_{x}^{i}, \quad \text { etc. }
\end{aligned}
$$

Then

$$
a^{i}=v^{i}-\bar{V}^{i}
$$

and applying (10) to (7), obtain

$$
\begin{aligned}
\mathrm{V}\left(\bar{x}_{i}+\delta_{x_{i}}, \bar{k}+\delta k, t_{i}\right)= & a^{i}+\bar{v}^{i}+v_{x}^{i} \delta x_{i}+v_{k}^{i} \delta k+\frac{1}{2} \delta x_{i}^{T} v_{x x}^{i} \delta x_{i} \\
& +\delta x_{i}^{T} v_{x k}^{i} \delta k+\frac{1}{2} \delta k^{T} v_{k k}^{i} \delta k+\ldots
\end{aligned}
$$

Similarly, expanding the quantity to be minimized in equation (6) about $\bar{x}_{i}, \bar{k}, \bar{x}_{i+1}, t$

$$
\begin{aligned}
L^{i} & +L_{x}^{i} \delta_{x_{i}}+\frac{1}{2} \delta_{x_{i}} L_{x x}^{i} \delta_{x_{i}}+a^{i+1}+\bar{V}^{i+1}+V_{x}^{i+1} \delta_{x_{i+1}}+V_{k}^{i+1} \delta k \\
& +\frac{1}{2} \delta_{x_{i+1}^{T}}^{T} V_{x x}^{i+1} \delta_{x_{i+1}}+\delta_{x_{i+1}^{T}}^{T} V_{x k}^{i+1} \delta k+\delta k^{T} V_{k k}^{i+1} \delta k+\ldots
\end{aligned}
$$

where, as above, $a^{i+1}+\bar{V}^{i+1}=v^{i+1}$.

Expression (12) is an infinite series in $\delta_{x_{i}}, \delta_{x_{i+1}}$ and $\delta_{k}$. But it is clear that there is a relationship between $\delta_{x_{i}}$ and $\delta_{x_{i+1}}$ through equation (1). This relationship may be used to eliminate either $\delta_{x_{i}}$ or $\delta x_{i+1}$ from (12), but to conform with equation (11), $\delta x_{i+1}$ will be removed.

$$
\begin{aligned}
& x_{i+1}=f\left(x_{i}, u_{i}, t_{i}\right) \\
& \bar{x}_{i+1}=f\left(\bar{x}_{i}, \bar{u}_{i}, t_{i}\right)
\end{aligned}
$$

$t_{L}$ and its derivatives are evaluated at $\bar{x}_{i}, u_{i}, t_{i}$. The control $u_{i}$ is
yet to be determined. 
Thus, $\delta_{x_{i+1}}=f\left(x_{i}, u_{i}, t_{i}\right)-f\left(\bar{x}_{i}, \bar{u}_{i}, t_{i}\right)$ or,

$$
\delta x_{i+1}=f\left(\bar{x}_{i}+\delta x_{i}, u_{i}, t_{i}\right)-f\left(\bar{x}_{i}, \bar{u}_{i}, t_{i}\right)
$$

In equation (13), $u_{i}$ is perfectly general. It will later be fixed by the minimization operation of equation (6).

Expanding (13) about $\bar{x}_{i}$, and defining

$$
\begin{aligned}
& f^{i}=f\left(\bar{x}_{i}, u_{i}, t_{i}\right) \\
& \bar{f}^{i}=f\left(\bar{x}_{i}, \bar{u}_{i}, t_{i}\right),
\end{aligned}
$$

obtain

$$
\delta x_{i+1}=\left(f^{i}-\bar{f}^{i}\right)+f_{x}^{i} \delta x_{i}+\frac{1}{2} \delta x_{i}^{T} f_{x x}^{i} \delta x_{i}+\ldots
$$

where the derivatives of $f^{i}$ are evaluated at $\left(\bar{x}_{i}, u_{i}, t_{i}\right)$.

Substituting (14) into (12), obtain

$$
\begin{aligned}
& L^{i}+a^{i+1}+\bar{V}^{i+1}+V_{x}^{i+1}\left(f^{i}-\bar{f}^{i}\right)+\frac{1}{2}\left(f^{i}-\bar{f}^{i}\right)^{T} V_{x x}^{i+1}\left(f^{i}-\bar{f}^{i}\right) \\
& +\left[L_{x}^{i}+v_{x}^{i+1} f_{x}^{i}+f_{x}^{i} V_{x x}^{i+1}\left(f^{i}-\bar{f}^{i}\right)\right] \delta_{x_{i}} \\
& +\left[V_{k}^{i+1}+\left(f^{i}-\bar{f}^{i}\right)^{T} V_{x k}^{i+1}\right] \delta k \\
& +\delta_{x_{i}}^{T} f_{x}^{i} V_{x k}^{i+1} \delta k \\
& +\frac{1}{2} \delta k^{T} v_{k k}^{i+1} \delta k \\
& +\frac{1}{2} \delta x_{i}^{T}\left[L_{x x}^{i}+V_{x}^{i+1} f_{x x}^{i}+f_{x}^{i} V_{x x}^{i+1} f_{x}^{i}+\left(f^{i}-\bar{f}^{i}\right)^{T} v_{x x}^{i+1} f_{x x}^{i}\right] \delta x_{i}+\ldots
\end{aligned}
$$

Recall that equation (5) has now been transformed to

$$
\text { "r.h.s. of equation }(11)=\min _{u_{i}}\{\operatorname{expression}(15)\} "
$$


As suggested earlier, the minimization in (16) may be performed in two stages.

First $u_{i}^{*}$ is found, which minimizes (15) with $\delta_{x_{i}}=0$ and $\delta_{k}=0$, i.e., $u_{i}^{*}$ minimizes

$$
L^{i}+a^{i+1}+\bar{V}^{i+1}+V_{x}^{i+1}\left(f^{i}-\bar{f}^{i}\right)+\frac{1}{2}\left(f^{i}-\bar{f}^{i}\right)^{T} V_{x x}^{i+1}\left(f^{i}-\bar{f}^{i}\right)+\ldots
$$

(The terms not printed in (17) are of third and higher order in $\left(f^{i}-\bar{f}^{i}\right)$, and thus are assumed negligible.)

For convenience, define

$$
H^{i}=H\left(\bar{x}_{i}, u_{i}^{*}, \bar{k}, t_{i}\right)=L^{i}+V_{x}^{i+1} f^{i}
$$

In (18), and for the rest of this paper, all functions of $u_{i}$ are evaluated at $u_{i}^{*}$.

Note that

$$
\begin{aligned}
& \mathrm{H}_{x}^{i}=L_{x}^{i}+V_{x}^{i+1} f_{x}^{i} \\
& H_{x x}^{i}=L_{x x}^{i}+V_{x}^{i+1} f_{x x}^{i} \text {, etc. }
\end{aligned}
$$

Since $(17)$ is at a minimum when evaluated at $u_{i}^{*}$, its first derivative with respect to $u_{i}$ must be zero;

$$
H_{u}^{i}+\left(f^{i}-\bar{f}^{i}\right)^{T} V_{x x}^{i+1} f_{u}^{i}=0
$$

In addition, the second derivative of (17) (to be defined as $\Delta$ ) must be positive definite at $u_{i}=u_{i}^{*}$;

$$
\Delta=H_{u u}^{i}+f_{u}^{i} v_{x x}^{i+1} f_{u}^{i}+\left(f^{i}-\bar{f}^{i}\right)^{T} v_{x x}^{i+1} f_{u u}^{i}>0
$$

(The third term in (20) does not appear in the 'weak variation' algorithms of $[4],[5],[9],[14])$. 
Expanding (15) about $u_{i}^{*}$, with $u_{i}=u_{i}^{*}+\delta u_{i}$, the following is obtained, using (19) and (20).

$$
\begin{aligned}
& L^{i}+a^{i+1}+\bar{V}^{i+1}+v_{x}^{i+1}\left(f^{i}-\bar{f}^{i}\right)+\frac{1}{2}\left(f^{i}-\bar{f}^{i}\right)^{T} v_{x x}^{i+1}\left(f^{i}-\bar{f}^{i}\right) \\
& +\left[H_{x}^{i}+f_{x}^{i} v_{x x}^{i+l}\left(f^{i}-\bar{f}^{i}\right)\right] \delta_{x} \\
& +\left[v_{k}^{i+1}+\left(f^{i}-\bar{f}^{i}\right)^{T} v_{x k}^{i+1}\right] \delta k \\
& +\delta_{x_{i}}^{T} f_{x}^{i} v_{x k}^{i+1} \delta k \\
& +\delta u_{i}^{T} f_{u}^{i^{T}} v_{x k}^{i+1} \delta k \\
& +\delta_{x_{i}}^{T}\left[H_{x u}^{i}+f_{x}^{i} v_{x x}^{i+1} f_{u}^{i}+\left(f^{i}-\bar{f}^{i}\right)^{T} v_{x x}^{i+1} f_{x u}^{i}\right] \delta u_{i} \\
& +\frac{1}{2} \delta x_{i}^{T}\left[H_{x x}^{i}+f_{x}^{i} V_{x x}^{i+1} f_{x}^{i}+\left(f^{i}-\bar{f}^{i}\right)^{T} V_{x x}^{i+1} f_{x x}^{i}\right] \delta x_{i} \\
& +\frac{1}{2} \delta k^{T} v_{k k}^{i+1} \delta k \\
& +\frac{1}{2} \delta u_{i}^{T} \Delta \delta u_{i}
\end{aligned}
$$

Terms of order $\left(\delta \mathrm{x}_{\mathrm{i}}\right)^{3},\left(\delta \mathrm{u}_{\mathrm{i}}\right)^{3},(\delta \mathrm{k})^{3}$ or $\mathrm{greater}$ have been ignored in $(21) . t$

The second stage of the minimization is accomplished when (21) is minimized with respect to $\delta_{u_{i}}$.

Taking the first derivative of (21) with respect to $\delta u_{i}$ and setting it to zero, obtain

$t_{\text {It }}$ is assumed that $\delta_{x_{i}}, \delta_{u_{i}}$ and $\delta k$ are small enough to justify this truncation.

$-8-$ 


$$
\delta_{u_{i}}=\beta_{1} \delta_{x_{i}}+\beta_{2} \delta \mathrm{k}
$$

where

$$
\begin{aligned}
& \beta_{1}=-\Delta^{-1}\left[\mathrm{H}_{\mathrm{ux}}^{\mathrm{i}}+\mathrm{f}_{\mathrm{u}}^{\mathrm{i}} \mathrm{V}_{\mathrm{xx}}^{\mathrm{i}+1} \mathrm{f}_{\mathrm{x}}^{\mathrm{i}}+\left(\mathrm{f}^{\mathrm{i}}-\bar{f}^{\mathrm{i}}\right)^{\mathrm{T}} \mathrm{v}_{\mathrm{xx}}^{\mathrm{i}+1} \mathrm{f}_{\mathrm{ux}}^{\mathrm{i}}\right] \\
& \beta_{2}=-\Delta^{-1} \mathrm{f}_{\mathrm{u}}^{\mathrm{T}} \mathrm{v}_{\mathrm{x} k \mathrm{k}}^{\mathrm{i}+1}
\end{aligned}
$$

Equation (22) is a linear feedback perturbation control law. It is sufficient to consider $\delta_{u_{i}}$ to be linear in $\delta_{x_{i}}$ and $\delta k$ because on substituting an expression of higher, order than (22) into (21), terms of higher order than quadratic would appear.

On substituting (22) into (21), the result is

$$
\begin{aligned}
& L^{i}+a^{i+1}+\bar{V}^{i+1}+V_{x}^{i+1}\left(f^{i}-\bar{f}^{i}\right)+\frac{1}{2}\left(f^{i}-\bar{f}^{i}\right)^{T} V_{x x}^{i+1}\left(f^{i}-\bar{f}^{i}\right) \\
& +\left[H_{x}^{i}+\left(f^{i}-\bar{f}^{i}\right)^{T} V_{x x}^{i+1} f_{x}^{i}\right] \delta_{x_{i}} \\
& +\left[V_{k}^{i+1}+\left(f^{i}-\bar{f}^{i}\right)^{T} V_{x k}^{i+1}\right] \delta k \\
& +\delta x_{i}^{T}\left[f_{x}^{i} V_{x k}^{i+1}-\beta_{1}^{T} \Delta \beta_{2}\right] \delta k \\
& +\frac{1}{2} \delta_{x_{i}^{T}}^{T}\left[H_{x x}^{i}+f_{x}^{i} V_{x x}^{i+1} f_{x}^{i}+\left(f^{i}-\bar{f}^{i}\right) V_{x x}^{i+1} f_{x x}^{i}-\beta_{1}^{T} \Delta \beta_{1}\right] \delta_{x_{i}} \\
& +\frac{1}{2} \delta k{ }_{k}^{T}\left[V_{k k}^{i+1}-\beta_{2}^{T} \Delta \beta_{2}\right] \delta k
\end{aligned}
$$

Expression (25) is the minimum of (15) with respect to $u_{i}$.

Thus, expression (25) is equal to the r.h.s. of equation (11), by (16). Therefore, coefficients of like powers of $\delta_{x_{i}}$ and $\delta k$ must be equal. 
Noting that

$$
\overline{\mathrm{V}}^{\mathrm{i}}=\overline{\mathrm{V}}^{\mathrm{i}+\mathrm{l}}+\overline{\mathrm{L}}^{\mathrm{i}},
$$

equating (11) and (25) produces the following difference equations, valid for $i=0, \ldots, N-1$.

$$
\begin{aligned}
& a^{i}=a^{i+1}+H^{i}-\bar{H}^{i}+\frac{1}{2}\left(f^{i}-\vec{f}^{i}\right)^{T} V_{x x}^{i+1}\left(f^{i}-\vec{f}^{i}\right) \\
& v_{x}^{i}=H_{x}^{i}+\left(f^{i}-\bar{f}^{i}\right)^{T} V_{x x}^{i+1} f_{x}^{i} \\
& v_{k}^{i}=V_{k}^{i+1}+\left(f^{i}-\bar{f}^{i}\right)^{T} V_{x k}^{i+1} \\
& v_{x k}^{i}=f_{x}^{i} V_{x k}^{i+1}-\beta_{1}^{T} \Delta \beta_{2} \\
& v_{k k}^{i}=V_{k k}^{i+1}-\beta_{2}^{T} \Delta \beta_{2} \\
& v_{x x}^{i}=H_{x x}^{i}+f_{x}^{i} V_{x x}^{i+1} f_{x}^{i}+\left(f^{i}-\bar{f}^{i}\right) V_{x x}^{i+1} f_{x x}^{i}-\beta_{1}^{T} \Delta \beta_{1}
\end{aligned}
$$

The boundary conditions are applied at $i=N$, and are the same as in [1]. They are found by expanding

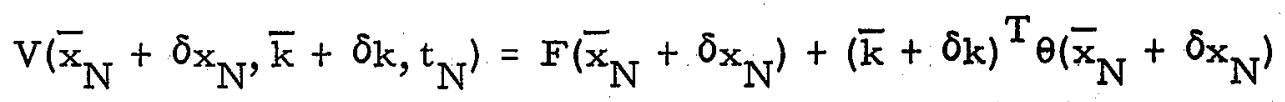

to second-order in a Taylor series in $\delta \mathrm{x}_{\mathrm{N}}$ and $\delta \mathrm{k}$. Because this is the last time step, $\overline{\mathrm{V}}^{\mathrm{N}}=\mathrm{V}^{\mathrm{N}}$. Thus,

$$
\mathrm{a}^{\mathrm{N}}=0
$$

and, from the expansion,

$$
\begin{aligned}
& v_{x}^{N}=F_{x}\left(\bar{x}_{N}\right)+\bar{k}^{T} \theta_{x}\left(\bar{x}_{N}\right) \\
& v_{k}^{N}=\theta^{T}\left(\bar{x}_{N}\right) \\
& v_{x k}^{N}=\theta_{x}^{T}\left(\bar{x}_{N}\right) \\
& v_{k k}^{N}=0
\end{aligned}
$$




$$
V_{x x}^{N}=F_{x x}\left(\bar{x}_{N}\right)+\bar{k}^{T} \theta_{x x}\left(\bar{x}_{N}\right)
$$

Thus, if we "integrate" equations (27)-(32) from $i=N-1$ to 0 with equations (33)-(38) as boundary conditions, then equations (19) and (22) show how to calculate $u_{i}=u_{i}^{*}+\delta u_{i}$ to get optimal improvement on performance index $\mathrm{V}\left(\mathrm{x}_{\mathrm{O}}, \mathrm{k}, \mathrm{t}_{\mathrm{o}}\right)$.

These results are only meaningful if the second-order truncations of the Taylor series above are good approximations of the full expansions. Thus $\delta_{x_{i}}, \delta_{x_{i+1}}, \delta k$, and $\delta_{u_{i}}$ must be small. There is no restriction on $\Delta u_{i}=u_{i}^{*}-\bar{u}_{i}$ except that $f^{i}-\bar{f}^{i}=f\left(\bar{x}_{i}, u_{i}^{*}, t_{i}\right)-f\left(\bar{x}_{i}, \bar{u}_{i}, t_{i}\right)$ must be sufficiently small to guarantee the smallness of $\delta_{x_{i+1}}$.

III. Comparison with and Extensions of Jacobson's Results III. 1. Comparison and Discussion

The case in which the discrete problem is an Euler discretization of a continuous problem is of interest. In that case,

$$
f\left(x_{i}, u_{i}, t_{i}\right)=x_{i}+\Delta t \tilde{f}\left(x_{i}, u_{i}, t_{i}\right)
$$

and

$$
L\left(x_{i}, u_{i}, t_{i}\right)=\tilde{L}\left(x_{i}, u_{i}, t_{i}\right) \Delta t
$$

Clearly,

$$
\dot{x}\left(t_{i}\right)=\lim _{\Delta t \rightarrow 0} \frac{x\left(t_{i}+\Delta t\right)-x\left(t_{i}\right)}{\Delta t}=\lim _{\Delta t \rightarrow 0} \frac{x_{i+1}-x_{i}}{\Delta t}=f\left(x_{i}, u_{i}, t_{i}\right)
$$

and

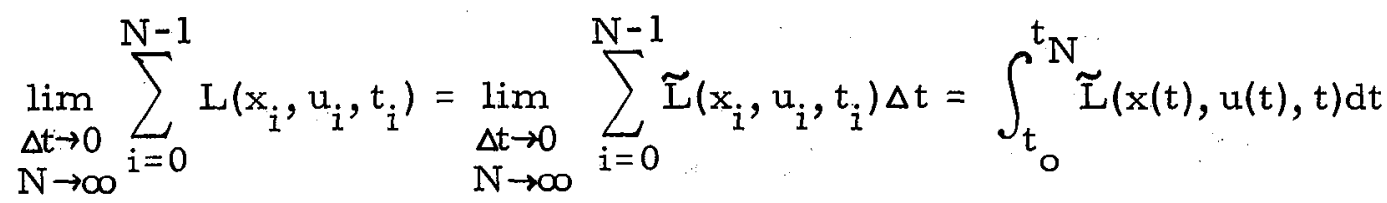

if the discretization is done with care. 
It is reasonable to expect that if the transformations (39) and (40) are applied to the results of the previous section and the limit is taken as $\Delta t \rightarrow 0$, equations should be obtained which solve the analogous continuous problem.

Jacobson [1] has solved that problem, and the statement of the problem, as well as the solution are reproduced below, in Appendix A.

Note that

$$
\mathrm{H}^{i}=\tilde{L}^{i} \Delta t+V_{x}^{i+1}\left(\bar{x}_{i}+\Delta t \tilde{f}^{i}\right)
$$

where the same abbreviated notation as in the last section is used.

Thus

$$
H^{i}=\left(\widetilde{L}^{i}+V_{x}^{i+l} \tilde{f}^{i}\right) \Delta t+V_{x}^{i+l} \bar{x}_{i}=\tilde{H}^{i} \Delta t+V_{x}^{i+1} \bar{x}_{i}
$$

Then, according to $(20)$

$$
\Delta=\widetilde{\mathrm{H}}_{u u}^{i} \Delta t+(\Delta t)^{2}\left[\tilde{f}_{u}^{i} v_{x x}^{i+1} \tilde{f}_{u}^{i}+\left(\tilde{f}^{i}-\overline{\tilde{f}}^{i}\right) v_{x x}^{i+1} \tilde{f}_{u u}^{i}\right]
$$

Define

$$
\widetilde{\Delta}=\Delta / \Delta t,
$$

which will be written

$$
\tilde{\Delta}=\widetilde{\mathrm{H}}_{\mathrm{uu}}^{\mathrm{i}}+\mathrm{A}^{\mathrm{i}} \Delta \mathrm{t}
$$

for clarity.

From (23) and (45),

$$
\beta_{1}=-\tilde{\Delta}^{-1}\left(\tilde{\mathrm{H}}_{u x}^{i}+\tilde{f}_{u}^{\mathrm{T}} V_{x x}^{i+1}\right)-\tilde{\Delta}^{-1}\left(\tilde{f}_{u}^{i^{T}} v_{x x}^{i+1} \tilde{f}_{x}^{i+1}+\left(\tilde{f}^{i}-\widetilde{f}^{i}\right)^{T} v_{x x}^{i+1} f_{u x}^{i}\right) \Delta t
$$

Similarly, from (24),

$$
\beta_{2}=-\tilde{\Delta}^{-1} \tilde{f}_{u}^{\sim_{u}^{i}} V_{x k}^{i+1}
$$

In the same manner, applying (39), (40), (43), (45), (47), and (48) to $(27)-(32)$, the following are simply obtained. 


$$
\begin{aligned}
& -\frac{a^{i+1}-a^{i}}{\Delta t}=\tilde{H}^{i}-\overline{\widetilde{H}}^{i}+\frac{1}{2}\left(\tilde{f}^{i}-\overline{\widetilde{f}}^{i}\right)^{T} V_{x x}^{i+1}\left(\tilde{f}^{i}-\overline{\widetilde{f}}^{i}\right) \Delta t \\
& -\frac{v_{x}^{i+1}-v_{x}^{i}}{\Delta t}=\tilde{H}_{x}^{i}+\left(\tilde{f}^{i}-\widetilde{\widetilde{f}}^{i}\right) V_{x x}^{i+1}+\left(\tilde{f}^{i}-\overline{\tilde{f}}^{i}\right)^{T} V_{x x}^{i+1} \tilde{f}_{\dot{x}}^{i} \Delta t \\
& -\frac{V_{k}^{i+1}-V_{k}^{i}}{\Delta t}=\left(\tilde{f}^{i}-\tilde{\sigma}^{i}\right)^{T} V_{x k}^{i+1} \\
& -\frac{\mathrm{v}_{\mathrm{xk}}^{\mathrm{i}+1}-\mathrm{v}_{\mathrm{xk}}^{\mathrm{i}}}{\Delta \mathrm{t}}=\underset{\mathrm{x}}{\stackrel{\mathrm{i}}{\mathrm{T}}} \mathrm{v}_{\mathrm{xk}}^{\mathrm{i}+1}-\beta_{1}^{\mathrm{T}} \tilde{\Delta} \beta_{2} \\
& -\frac{v_{k k}^{i+1}-v_{k k}^{i}}{\Delta t}=-\beta_{2}^{T} \tilde{\Delta} \beta_{2} \\
& -\frac{V_{x x}^{i+1}-v_{x x}^{i}}{\Delta t}=\tilde{H}_{x x}^{i}+\tilde{f}_{x}^{i} V_{x x}^{i+1}+V_{x x}^{i+1} \tilde{f}_{x}^{i}+\beta_{1}^{T} \tilde{\Delta} \beta_{1} \\
& +\Delta t\left[\tilde{f}_{x}^{i} v_{x x}^{i+1} \tilde{f}_{x}^{i}+\left(\tilde{f} \tilde{f}^{i}-\bar{f}_{i}^{i}\right)^{T} v_{x x}^{i+1} \tilde{f}_{x x}^{i}\right]
\end{aligned}
$$

Jaçobson's [1] equations for $\beta_{1}, \beta_{2}, a, v_{x}, v_{k}, v_{x k}, v_{k k k}$, and $\mathrm{V}_{\mathrm{xx}}$ ane reproduced below in Appendix A. Inspection will reveal agreement between those and (47)-(54) as $\Delta t \rightarrow 0$.

It should be noted that although the discrete $f, L$, and $H$ are related to their respective continuous counterparts through (39), (40), and (43), the discrete $a, \bar{v}$, derivatives of $v, \beta_{1}$, and $\beta_{2}$ directly approximate the continuous quantities. As $\Delta t \rightarrow 0$, the discrete and continuous versions of the latter quantities approach one another.

Equations (39) and (40) and the transformations that resulted from them were used to show the connection between the present discrete equations and the earlier [1] continuous equations. However, cases may exist where (39) and (40) are useful numerical methods with which to solve a continuous problem. ${ }^{+}$Then, (47)-(54) contain

† Continuous-time problems which are particularly sensitive to $u$ may require a large number of small time steps when the algorithms of [1], [2] are used. Then, since $\Delta t$ is small, sufficient integration accuracy may be obtained from an Euler scheme. See [2, page 17]. 
the full dependence on $\Delta t$, which involves terms of order $\Delta t$ and higher. It may be worth while to retain high order terms [14].

Also, (47)-(54) indicate that some of the arguments of the right sides are to be evaluated at time $i+1$, and others must be evaluated at time i. A simple Euler discretization of the continuous time algorithm [1], [2] would evaluate all arguments at time $i+1$.

It may be possible to obtain more useful versions of (47)-(54) by replacing (39) and (40), the Euler discretizations of $f$ and $L$, by a more sophisticated, accurate scheme.

III. 2. Description of the Algorithm

The discrete algorithm is very similar to the continuous algorithm [1, section 4.8], and is outlined in Flow Chart II.

The algorithm is a successive approximation process, and each approximation has two stages. In the first stage, $k$ is kept constant, and optimization takes place with respect to $u_{i}$, without regard to the value of $\theta$. In the second, $\delta \mathrm{k}$ is calculated to reduce $\theta$ in absolute value.

The first stage proceeds as follows. Equation (1) is "integrated" using initial conditions $x_{0}$ and nominal control $\bar{u}_{0}, \ldots, \bar{u}_{N-1}$. Then equations (27), (28), and (32) are integrated back from $i=N$, with boundary conditions (33), (34), and (38).

If $\mathrm{a}^{\circ}$ is not close to zero, then, by definition $(10)$, the nominal control is not close to optimal for the current value of $\bar{k}$. To improve the trajectory (i.e., to get closer to the optimal and reduce a ${ }^{\circ}$ ), (19) is solved for $u_{i}^{*}$ and $(22)$ is used to calculate $u_{i}=u_{i}^{*}+\delta u_{i}$, which is used as the new optimal control in (1). The cycle repeats. If necessary (see below for the descriptions of the tests to explain this 


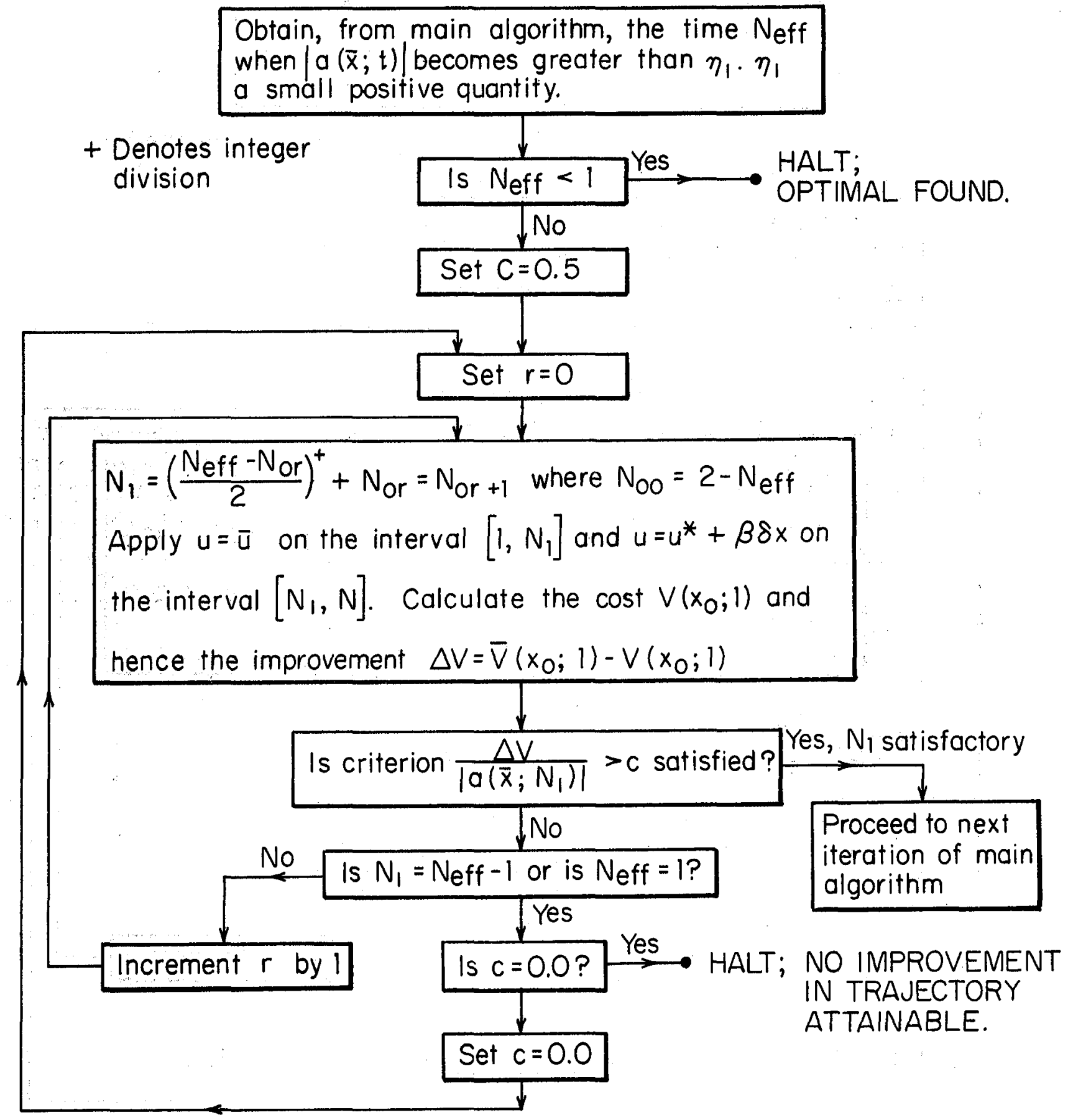

FLOWCHART I: "STEP SIZE ADJUSTMENT METHOD". 


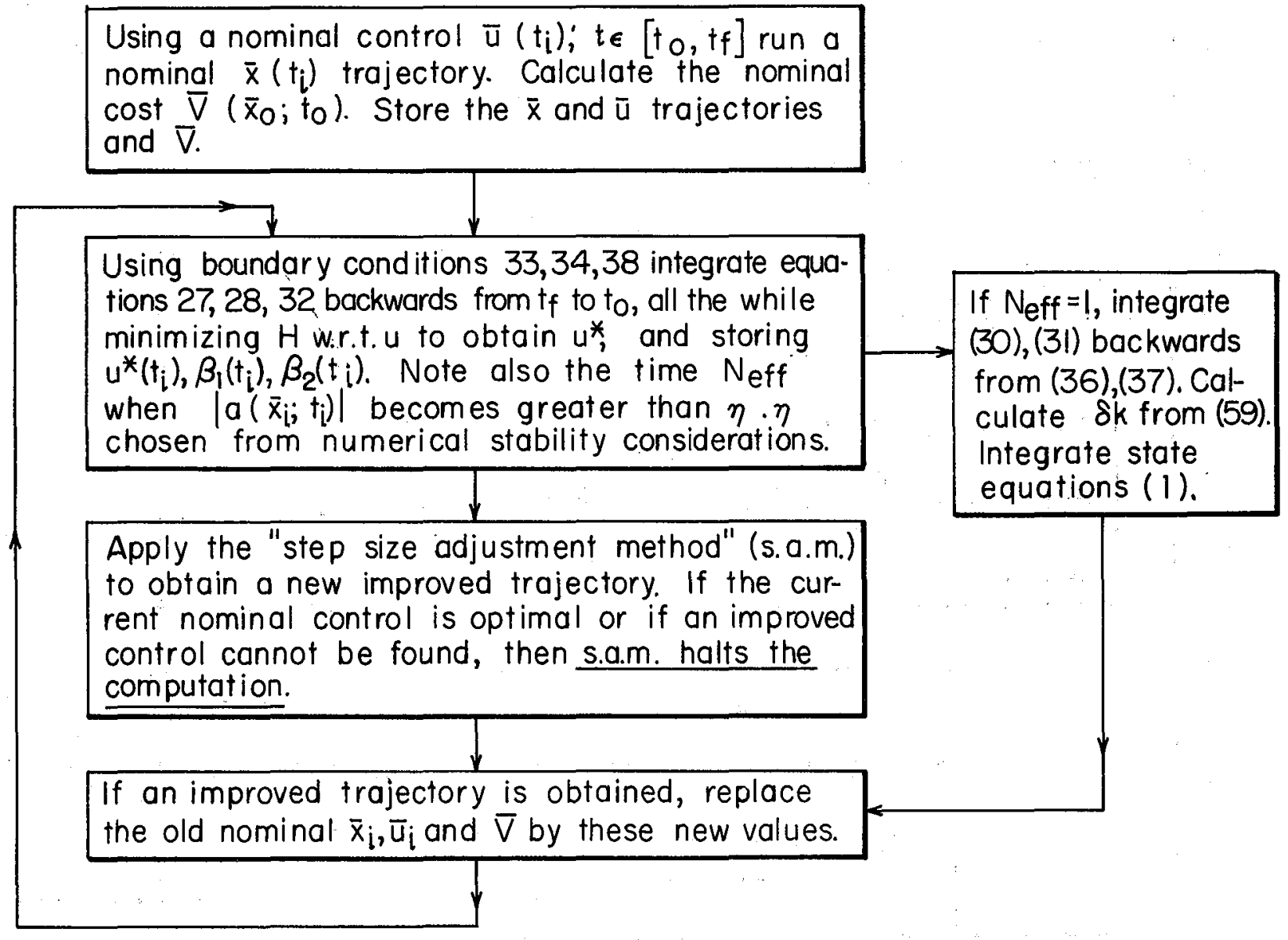

FLOW CHART II: THE OVERALL COMPUTATIONAL PROCEDURE 
necessity) the step-size adjustment routine is called. (This routine will not be discussed here, but, for completeness, it appears schematically in Flow Chart I. It is described in the references, [1], and $[2$, section 4$]$.)

If $\mathrm{a}^{\circ}$ is close to zero, and $\theta$ is also close to zero, the problem is solved.

If $\mathrm{a}^{\circ}$ is close to zero but $\theta$ is not, the algorithm enters its second stage: $k$ is modified (according to the formula of the next section) to reduce each component of $\theta$ in absolute value.

III. 3. Determination of $\delta \mathrm{k}$

Ok is found in the following manner. Jacobson has shown [1, section 4.6$]$ that, to second-order, the proper value of $\delta \mathrm{k}$ is that which maximizes $V\left(\bar{x}_{0}, \bar{k}+\delta_{k}, t_{0}\right) \cdot{ }^{+}$But

$$
\mathrm{V}\left(\bar{x}_{\circ}, \bar{k}+\delta k, t_{o}\right)=a^{o}+\bar{v}^{\circ}+V_{k}^{\circ} \delta k+\frac{1}{2} \delta k^{T} v_{k k}^{o} \delta k
$$

Therefore the proper value of $\delta \mathrm{k}$ satisfies

$$
\mathrm{v}_{\mathrm{k}}^{\mathrm{O}}+\mathrm{v}_{\mathrm{kk}}^{\mathrm{O}} \delta \mathrm{k}=0
$$

or

$$
\delta k^{T}=-v_{k k}^{o-1} v_{k}^{o^{T}}
$$

(Jacobson shows that $V_{\mathrm{kk}}^{\circ}$ is negative definite, so that $\mathrm{V}_{\mathrm{kk}}^{\circ}{ }^{-1}$ exists:)

Since, in the present algorithm, $\delta \mathrm{k}$ is only evaluated when $f^{i}-\bar{f}^{i}=0$ (because $\left.a^{0}=0\right), V_{k}^{o}=\theta^{T}\left(\bar{x}_{N}\right)$ from equations (29) and (35).

Then, (57) becomes

$$
\delta_{k}=-v_{k k}^{o-1} \theta\left(\bar{x}_{N}\right)
$$

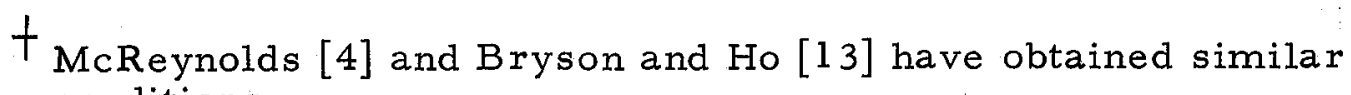
conditions.

† Provided that the linearised system is controllable, and $\theta_{x}^{T}$ has full rank. 
Following [1], $\mathrm{k}$ is modified according to $(58) ;(1)$ is then integrated forward with $u_{i}=u_{i}^{*}+\delta u_{i}$ chosen according to (19) and (22). If the resultant value of $\theta\left(x_{N}\right)$ is not smaller in absolute value (component-wise) than $\theta\left(\bar{x}_{N}\right)$, choose

$$
\delta \mathrm{k}=-\epsilon \mathrm{V}_{\mathrm{kk}}^{\mathrm{o}} \theta\left(\overline{\mathrm{x}}_{\mathrm{N}}\right)
$$

where $0<\epsilon<1$, and reduce $\epsilon$ until $\theta\left(x_{N}\right)$ is reduced and $a^{0}$ is near zero.

\section{4. New Criteria}

It is essential that $\delta_{x_{i}}$ and $\delta_{k}$ be kept small. This ensures that $\delta u_{i}$ will be small, and thus the second-order expansions of (6) will remain valid. If $\delta_{x_{i}}$ and $\delta k_{k}$ are found to be too large, i.e., if they invalidate the truncations of the Taylor series in section II, means for reducing them are presented in Jacobson's algorithms [1, section 4.2.1], [1, section 4.8], [2, section 4]. These techniques apply to the discrete problem as well as to the continuous.

There are criteria in [1] and [2] for deciding whether to reduce $\delta_{x_{i}}$ and $\delta_{k}$ or not. However, an addition criterion, required for fixed end point problems is described below (Test 1).

A criterion, alternative to that in [1], [2] is also given. This criterion (Test 2) is useful in cases where it is desirable to keep the 'new trajectory' in the immediate neighborhood of the nominal. + Test 1

Although $\delta_{k}$ is chosen according to (59) (where $\epsilon$ is such that $\theta\left(x_{N}\right)$ is reduced), it may lie outside the range of validity of the expansion (11) (when truncated at second-order terms).

t Such may be the case when the trajectory must be prevented from "jumping" to another near by local minimum. In the following section, an example is discussed in detail where this was found to be necessary. 
At $i=0,(11)$ coincides with (5.5). Since both sides of (55) may be independently measured (i.e., choose $\delta_{k}$ and evaluate the left-hand side. Then integrate (1) as described above and evaluate the right-hand side, $\left.\mathrm{V}\left(\overline{\mathrm{x}}_{\mathrm{o}}, \overline{\mathrm{k}}+\delta_{\mathrm{k}, \mathrm{t}_{\mathrm{o}}}\right)\right),(55)$ may be considered to be a test of $\delta_{\mathrm{k}}$.

If $\delta_{k}$ is given by (59), then (55) predicts that

$$
\mathrm{V}\left(\overline{\mathrm{x}}_{\mathrm{o}}, \overline{\mathrm{k}}+\delta \mathrm{k}, \mathrm{t}_{\mathrm{o}}\right)-\overline{\mathrm{V}}^{\mathrm{O}}=\mathrm{a}^{\mathrm{o}}-\left(\epsilon-\frac{1}{2} \epsilon^{2}\right) \theta^{\mathrm{T}}\left(\overline{\mathrm{x}}_{\mathrm{N}}\right) \mathrm{V}_{\mathrm{kk}}^{\mathrm{o}} \theta\left(\overline{\mathrm{x}}_{\mathrm{N}}\right)
$$

If $(60)$ does not predict the change in $V$ to within a given tolerance, then $\epsilon$ should be reduced until it does.

\section{Test 2}

From (4) and (9),

$$
\begin{aligned}
& V^{i}=\sum_{j=i}^{N} L^{j}+F\left(x_{N}\right)+k^{T} \theta\left(x_{N}\right) \\
& \bar{V}^{i}=\sum_{j=i}^{N} \bar{L}^{j}+F\left(\bar{x}_{N}\right)+\bar{k}^{T} \theta\left(\bar{x}_{N}\right)
\end{aligned}
$$

Thus

$$
\delta v^{i}=V^{i}-\bar{v}^{i}=\sum_{j=i}^{N} \delta L^{j}+\left(F\left(x_{N}\right)-F\left(\bar{x}_{N}\right)\right)+\left(k^{T} \theta\left(x_{N}\right)-\bar{k}_{\theta}^{T}\left(\bar{x}_{N}\right)\right)
$$

But, from (11),

$$
\delta v^{i}=a^{i}+v_{x}^{i} \delta x_{i}+v_{k}^{i} \delta k+\frac{1}{2} \delta x_{i}^{T} v_{x x}^{i} \delta x_{i}+\delta x_{i}^{T} v_{x k}^{i} \delta k+\frac{1}{2} \delta k^{T} v_{k k}^{i} \delta k
$$

Since (63) and (64) must be equal, their proximity is a test on the size of $\delta_{x_{i}}$ and $\delta_{k}$. This is because (63) is an exact expression, and (64) is an approximation dependent on $\delta_{x_{i}}$ and $\delta k$.

In order to use (63) and (64) as a step-by-step test of $\delta_{x_{i}}$, their form should be modified. This is because (63) involves $x_{N}$, which is not yet available at step $i$ of the forward integration. The modification 
is a simple one: from (63),

$$
\delta V^{o}=\sum_{j=0}^{N} \delta L^{j}+\left(F\left(x_{N}\right)-F\left(\bar{x}_{N}\right)\right)+\left(k^{T} \theta\left(x_{N}\right)-\bar{k}^{T} \theta\left(\bar{x}_{N}\right)\right)
$$

Thus,

$$
\delta V^{i}-\delta V^{o}=\sum_{j=0}^{i-1} \delta L^{j}
$$

Similarly, $\delta \mathrm{V}^{\mathrm{i}}-\delta \mathrm{V}^{\circ}$ may be calculated from (64).

$$
\begin{aligned}
\delta v^{i}-\delta v^{\circ}= & {\left[a^{i}+v_{x}^{i} \delta_{x_{i}}+v_{k}^{i} \delta k+\frac{1}{2} \delta x_{i}^{T} v_{x x}^{i} \delta x_{i}+\delta x_{i}^{T} v_{x k}^{i} \delta k\right.} \\
& \left.+\frac{1}{2} \delta k^{T} v_{k k}^{i} \delta k\right]-\left[a^{o}+v_{k}^{o} \delta k+\frac{1}{2} \delta k^{T} v_{k k}^{o} \delta k\right]
\end{aligned}
$$

The last equation may be simplified somewhat by noticing that $\mathrm{V}_{\mathrm{k}}^{\mathrm{i}}=\mathrm{V}_{\mathrm{k}}^{\mathrm{O}}$ whenever $\delta \mathrm{k}$ is evaluated. Thus

$$
\begin{aligned}
\delta v^{i}-\delta v^{\circ}= & a^{i}-a^{o}+v_{x}^{i} \delta_{x_{i}}+\frac{1}{2} \delta_{x_{i}}^{T} v_{k x}^{i} \delta_{x_{i}}+\delta x_{i}^{T} v_{x k}^{i} \delta k \\
& +\frac{1}{2} \delta k^{T} v_{k k}^{i} \delta k-\frac{1}{2} \delta k^{T} v_{k k}^{o} \delta k
\end{aligned}
$$

Then, test 2 is performed by determining whether (66) agrees with (68) within a given tolerance. If the test is failed then $\delta \mathrm{k}$ should be reduced, or, if $\delta k$ is not present, $\delta_{x_{i}}$ should be reduced by the step-size adjustment method.

This test is particularly simple to apply in cases where $L\left(x_{i}, u_{i}, t_{i}\right) \equiv 0$

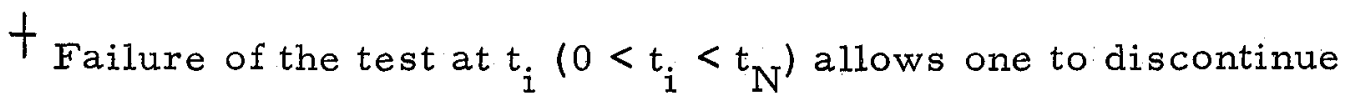
integration of this 'trial trajectory' at $t_{i}$ instead of integrating all the way to $t_{N}$; this can save considerable computer time.
} 
IV. Numerical Example - Comparison with McReynolds' Successive Sweep Method

IV. 1. Statement of the Orbit Transfer Problem

An orbit transfer problem [4], [5], [7], [8], [12] has been solved. In this problem, a control sequence must be found to maximize the radial distance of a rocket from the sun, with the terminal condition that the rocket be in a solar orbit.

$\mathbf{x}_{\mathbf{i}}$ is a 3-vector, whose components represent radial distance (from the sun), radial velocity, and angular velocity, respectively, normalized so that the initial condition (in earth's orbit) is

$$
\begin{aligned}
& x_{0}=\left(\begin{array}{l}
1 \\
0 \\
1
\end{array}\right) \\
& \theta\left(x_{N}\right)=\left(\begin{array}{l}
x_{2, N} \\
x_{3, N}-\frac{1}{\sqrt{x_{1}, N}}
\end{array}\right) ; \quad(\theta=0 \text { is the condition for } a
\end{aligned}
$$

state to be in a stable orbit.)

$\widetilde{L}^{i}=0$.

$F\left(x_{N}\right)=x_{1, N} \cdot$ Thus,

$\mathrm{V}=\mathrm{x}_{1, \mathrm{~N}}+\mathrm{k}_{1} \theta_{1}+\mathrm{k}_{2} \theta_{2}$

$\tilde{\tilde{f}}^{i}=\left(\begin{array}{l}\mathrm{x}_{2, i} \\ \frac{\mathrm{x}_{3, i}^{2}}{\mathrm{x}_{1, i}}-\frac{1}{x_{1, i}^{2}}+A^{i} \sin u_{i} \\ -\frac{x_{2, i} x_{3, i}}{x_{1, i}}+A^{i} \cos u_{i}\end{array}\right)$ 
where

$$
A^{i}=\frac{.1405}{1.0-.07487 \varepsilon_{i}}
$$

The time interval $\left[0, t_{N}\right]$ is given.

Note that $\tilde{f}^{i}\left(x_{i}, u_{i}\right)=\tilde{F}\left(x_{i}\right)+G^{i}\left(u_{i}\right)$. Thus $\tilde{H}^{i}=v_{x}^{i+1}\left(\widetilde{F}\left(x_{i}\right)+G^{i}\left(u_{i}\right)\right)$ and $\tilde{H}_{u x}^{i}$ and $\tilde{f}_{u x}^{i}$ vanish.

This statement of the problem was inserted into (46)-(54) with terms of order higher than $\Delta t$ dropped. The equations become:

$$
\begin{aligned}
& \tilde{\Delta}=\tilde{\mathrm{H}}_{u \mathrm{u}}^{\mathrm{i}}=\mathrm{V}_{\mathrm{x}}^{\mathrm{i}+1} \mathrm{G}_{\mathrm{uu}}^{\mathrm{i}} \\
& \beta_{1}=-\tilde{\Delta}^{-1} \tilde{\tilde{f}}_{\mathrm{u}}^{\mathrm{N}} \mathrm{V}_{\mathrm{xx}}^{\mathrm{i}+1} \\
& \beta_{2}=-\tilde{\Delta}^{-1} \tilde{f}_{\mathrm{u}}^{\sim_{\mathrm{j}}^{T}} \mathrm{~V}_{\mathrm{xk}}^{\mathrm{i}+1}
\end{aligned}
$$$$
a^{i}=a^{i+1}+V_{x}^{i+1}\left(G^{i}\left(u_{i}^{*}\right)-G^{i}\left(\bar{u}_{i}\right)\right) \Delta t
$$

$$
v_{x}^{i}=v_{x}^{i+1}+\left(V_{x}^{i+1} \tilde{F}_{x}\left(\bar{x}_{i}\right)+\left(G^{i}\left(u_{i}^{*}\right)-G^{i}\left(\bar{u}_{i}\right)\right) v_{x x}^{i+1}\right) \Delta t
$$$$
v_{k}^{i}=V_{k}^{i+1}+\left(G^{i}\left(u_{i}^{*}\right)-G^{i}\left(\bar{u}_{i}\right)\right) V_{x k}^{i+1} \Delta t
$$$$
\mathrm{V}_{x k}^{\mathrm{i}}=\mathrm{V}_{\mathrm{xk}}^{\mathrm{i}+1}+\left(\tilde{\mathrm{F}}_{\mathrm{x}}\left(\bar{x}_{\mathrm{i}}\right) \mathrm{V}_{\mathrm{xk}}^{\mathrm{i}+1}-\beta_{1}^{\mathrm{T}} \tilde{\Delta}_{2}\right) \Delta t
$$$$
\mathrm{V}_{\mathrm{kk}}^{\mathrm{i}}=\mathrm{V}_{\mathrm{kk}}^{\mathrm{i}+1}-\beta_{2}^{\mathrm{T}} \tilde{\Delta}_{2} \Delta \mathrm{t}
$$$$
V_{x x}^{i}=V_{x x}^{i+1}+\left\{V_{x}^{i+1} \tilde{F}_{x x}\left(\bar{x}_{i}\right)+\widetilde{F}_{x}\left(\bar{x}_{i}^{T}\right) V_{x x}^{i+1}+V_{x x}^{i+1} \widetilde{F}_{x}\left(\bar{x}_{i}\right)+\beta_{1}^{T} \tilde{\Delta}_{1}\right\} \Delta t
$$

where $u_{i}^{*}$ was found by maximizing $\tilde{H}^{i}$ which was equivalent to maximizing $v_{x}^{i+1} G^{i}\left(u_{i}\right)$, which, in turn, was equivalent to finding the maximum of

$$
v_{x, 2}^{i+1} \sin u_{i}+v_{x, 3}^{i+1} \cos u_{i} \text {. }
$$


Thus

$$
v_{x, 2}^{i+1} \cos u_{i}^{*}-v_{x, 3}^{i+1} \sin u_{i}^{* *}=0
$$

or,

$$
u_{i}^{* *}=\arctan \left(V_{x, 2}^{i+1} / v_{x, 3}^{i+1}\right)
$$

Terms of higher order in $\Delta t$ were dropped on the assumption that such terms were negligible in comparison with those of order $\Delta t$.

In the forward integration phases, $u_{i}=u_{i}^{*}+\delta_{u_{i}}$ was computed directly by màximizing

$$
\begin{aligned}
& \tilde{H}^{i}\left(\bar{x}_{i}+\delta x_{i}, u_{i}, v_{x}^{i+1}+\delta x_{i+1}^{T} v_{x x}^{i+1}+\delta k^{T} V_{k x}^{i+1}\right) \\
& =\left(V_{x}^{i+1}+\delta x_{i+1}^{T} V_{x x}^{i+1}+\delta k^{T} V_{k x}^{i+1}\right)\left(\tilde{F}\left(\bar{x}_{i}+\delta x_{i}\right)+G^{i}\left(u_{i}\right)\right)
\end{aligned}
$$

with respect to $u_{i}$. Note that $\delta_{x_{i+1}}$ should be replaced by (14), which becomes

$$
\delta_{x_{i}+1}=\delta_{x_{i}}+\Delta t\left[\left(G\left(u_{i}\right)-G\left(\bar{u}_{i}\right)\right)+F_{x}\left(\bar{x}_{i}\right) \delta_{x_{i}}+\frac{1}{2} \delta_{x_{i}}^{T} F_{x x}\left(\bar{x}_{i}\right) \delta_{x_{i}}\right]
$$

However, this is of nigher order than the degree of approximation, and it is satisfactory to replace $\delta_{x_{i+1}}$ in (79) by $\delta_{x_{i}}$.

The new criteria described in the previous section were experimentally applied. Test 1 appeared to be essential for the algorithm to converge. Without it, $\delta \mathrm{k}$ was often chosen too large. Test 2 was found to be helpful and time saving. A more detailed discussion will be found in section $V$.

IV. 2. Comparison with Successive Sweep Method

This algorithm converges somewhat faster than McReynolds' Successive Sweep Method [4], [5], [6] on this problem, starting from the same initial nominal. This may be because the two techniques 
differ primarily in the minimization $\neq$ and $f^{i}-\bar{f}^{i}$ and $H^{i}-\bar{H}^{i}$ terms which are present here and absent from the successive sweep method. But, close to the optimal, those terms are small, and the minimization yields results which are close to McReynolds' method for choosing $\delta u_{i}$. Thus, close to the optimal, the algorithms are very nearly the same. Earlier in the computation, the terms are large, and the minimization permits the present routine to take larger steps. Thus, this routine is able to get to the vicinity of the nominal in fewer iterations than the Successive Sweep Method, and once there, to take just as many additional iterations to converge.

In addition, this routine does not evaluate $H_{u u}$ (or $\Delta$ ) until after a minimization has been performed. Thus $\mathrm{H}_{\mathrm{uu}}$ is always negative (definite). McReynolds evaluates $\mathrm{H}_{\text {uu }}$ on the nominal trajectory, and so, he must either choose his initial nominal so that $\mathrm{H}_{\mathrm{uu}}$ is negative, or he must invoke a device to partially overcome the difficulty. ${ }^{t}$

V. Numerical Results

V.I. Discussion of the Trajectories in Tables 1-4

Tables 1 - 3 contain optimal trajectories calculated for the problem of the previous section by means of the algorithm described above. (The computer program is presented in detail in Appendix B. See the section on the BETA subroutine for an explanation of $\beta_{1}, \beta_{2}, \beta_{3}$.)

The value of 3.32 was used for $t_{N}$ in order to compare results with [4] and [5]. The other value, 3. 3194 was determined in [12], where the authors solved a minimum time problem. Their problem

$\overline{t-\left|\mathrm{H}_{u u}^{i}+B^{i}\right| \text { is used in place of } H_{u u}^{i}}$ where $B^{i}$ is chosen to go to zero as the nominal is approached. See [5, page 596].

$\neq$ Which becomes a maximization in this problem. 
was identical with the present problem, except that they specified $\mathrm{x}_{1}\left(\mathrm{t}_{\mathrm{N}}\right)=1.525$ (corresponding to the orbit of Mars) as a constraint and left $t_{N}$ free. Our results agree most closely with those of [12]. (The normalized values of $V_{x}^{O}$ agree with $\lambda\left(t_{0}\right)$ given in $[12]$, to 3 figures.)

The rather large differences between the results of 100 time steps and of 400 steps indicate that 100 "Euler integration" steps are not really sufficient to model the continuous time dynamic system. It should be noted that the greatest discrepancies occur in the secondorder quantities. But from (69)-(78), those quantities are the only ones whose exact equations have high order $\Delta t$ terms near the nominal. (Near the nominal, $f^{i}-\bar{f}^{i}$ is small or zero.) This may account for the difference in values between our $\beta_{1}, \beta_{2}$, and $\beta_{3}$ and those given by McReynolds [5].

It is interesting to note that many different attempts have been made to solve this problem [4], [5], [7], [8], [12]. Our results agree most closely with those quoted in [12] and are more detailed than those previously published.

Table 4 contains a trajectory which maximizes $V$ without regard to terminal constraints for nearly optimal values of $k_{1}$ and $k_{2}$. It is interesting to note that the maximum obtained for $V$ is far from the maximum $\mathrm{V}$ obtained in Tables $1-3$, and the $\theta^{\prime} \mathrm{s}$ are not zero. Thus the free end point problem, with $k_{1}$ and $k_{2}$ set to their optimal values has at least two local maxima; the one maximum coincides with the point $\theta=0$, while the other does not. (We have found that if, starting with this other maximum solution, and the optimal $\mathrm{k}^{\prime} \mathrm{s}$, the $k^{\prime}$ s are changed successively to reduce $|\theta|$, using the algorithm, 
then the optimal solution to the problem is obtained. I. e. the $k^{\prime}$ sare adjusted away from their 'optimal' values, but again return to these optimal values, at which stage the 'correct' minimum of $\mathrm{V}$ is attained and $\theta=0$.)

On the average, the program took approximately 3 seconds per iteration for the 100 step program and 12 seconds per iteration for the 400 step program. For this purpose, the "number of iterations" is defined as the number of times the program went into BAKINT (see Appendix B) i.e., the number of times (27), (28), and (32) were integrated. Thus, an iteration includes at least one but posibibly as many as 9 times through FORINT, the subprogram that integrates the state equations (1) forward. Also, an iteration may include DKCALC, the program to integrate (30) and (31) and calculate $\delta \mathrm{k}$ by $(59)$.

In the earlier versions of the program, where Test 2 was absent, iteration times averaged as much as 6 seconds for 100 step trajectories. More details on this follow.

The nominal used to compute the trajectory in Table 1 was the nominal McReynolds used: $\overline{\mathrm{k}}_{1}=-1 ; \overline{\mathrm{k}}_{2}=1 ; \bar{u}(\mathrm{t})=1.57078$ for $0 \leqslant t \leqslant 1.66 ; \bar{u}(t)=5.7124$ for $1.66<t<3.32$. Convergence to $\left|\theta_{i}\left(x_{N}\right)\right|<10^{-6}(i=1,2)$ required 15 iterations.

The control history of the nominal used for Tables 2 and 3 was the optimal trajectory computed in [5]. (It was linearly interpolated to 100 points, and then expanded to 400 points by repeating each value four times). For Table $2, \bar{k}_{1}=-1.41936541, \bar{k}_{2}=1.264609$, and convergence required 10 iterations. For Table $3, \bar{k}_{1}=-1.399631$, $\overline{\mathrm{k}}_{2}=1.260031$ (optimal values from $[4]$ ), and 11 iterations were required. 
Table 4 was started from a nominal consisting of the control

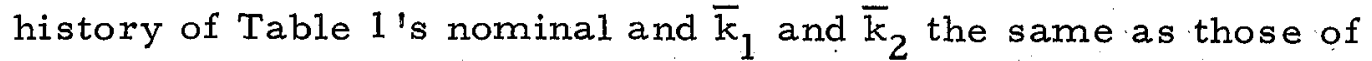
Table 3. It took 6 iterations to "converge." V.2. Uses of Tests 1 and 2

With neither Test 1 nor Test 2 present the algorithm did not converge. Constraining each new trajectory by the requirement that Test 1 be satisfied was sufficient to ensure convergence. Because this constraint was usually effective - i.e., many values of $\delta \mathrm{k}$ were rejected--this problem appears to be very sensitive to changes in the multipliers $k$ 。

Pairs of runs were compared: of each, one had only Test 1; the other had both tests. The comparison indicated a certain redundancy between the two tests. A large number of trial $\delta_{k}$ 's were rejected by both Test 1 (where that was the only test) and Test 2 (where both tests existed.) In fact, the same values of $\delta \mathrm{k}$ were ultimately accepted by the two programs, and the programs generally converged to the same optimal trajectory in the same number of steps.

However, the redundancy was not complete. There were $\delta k^{\prime} \mathrm{s}$ that were accepted by Test 2 and rejected by Test 1 .

But the redundancy is helpful. Test 2 can be invoked often in the forward integration phase, while Test 1 can only be invoked after the forward integration phase is complete. Thus Test 2 can save execution time. This time appears to be quite significant: with both tests present, a 100 step iteration took about 3 seconds. With only Test 1 , a 100 step iteration took - on the average - more than six seconds. (As pointed out in the footnote on page 20, the forward integration of the system equations can be terminated as soon as 
Test 2 fails. However, Test 1 requires that the integration be performed up until $t_{N}$. This accounts for the 'time saving' when Test 2 is included.)

A difficulty was encountered in using the tests. As the algorithm approached the optimal, steps and changes in parameters tended to grow rather small. Then all tests which involve differences of large quantities become less reliable - in fact, excessively conservative. Thus there should be some means of disabling the tests when $\delta_{x_{i}}$ or $\delta_{k}$ are sufficiently small.

Once the difficulty was recognized, Test 1 was disabled when $\delta \mathrm{V}^{\circ}=\mathrm{V}^{\circ}-\overline{\mathrm{V}}^{\circ}$ was less, in absolute value, than $10^{-6} \overline{\mathrm{V}}^{\circ}$. Test 2 was disabled when the absolute value of

$$
\mathrm{a}^{\circ}+\mathrm{V}_{\mathrm{k}}^{\mathrm{O}} \delta \mathrm{k}+\frac{1}{2} \delta \mathrm{k}^{\mathrm{T}} \mathrm{V}_{\mathrm{kk}}^{\mathrm{o}} \delta \mathrm{k}
$$

was less than $10^{-6} \overline{\mathrm{V}}^{\circ}$.

V. 3. Behavior of the Algorithm

The existence of the maximum in Table 4 may be illustrated by analogy with a static maximization of a function of a single variable. See figure 1.

In order to maximize $\mathrm{V}(\mathrm{u})$, one may approximate $\mathrm{V}$ with a second-order Taylor expansion in the neighborhood of $\bar{u}$, a nominal value.

$$
V(u) \approx V(\bar{u})+V^{\prime}(\bar{u})(u-\bar{u})+\frac{1}{2} V^{\prime \prime}(\bar{u})(u-\bar{u})^{2}
$$

The value of $u$ that maximizes this is given by

$$
0=V^{\prime}(\bar{u})+\frac{1}{2} V^{\prime \prime}(\bar{u})(u-\bar{u})
$$

or 


$$
u=\bar{u}-\frac{V^{\prime}(\bar{u})}{V^{\prime \prime}(\bar{u})}
$$

Equation (81) may be used to predict the improvement in $V$ using $(82)$.

$$
V(u)-V(\bar{u})=-\frac{1}{2} \frac{V^{\prime}(\bar{u})^{2}}{V^{\prime \prime}(\bar{u})}
$$

which is positive for $V^{\prime \prime}(u)<0$. Then (83) may be used as a criterion for optimality: when (83) is zero, $\bar{u}$ is a maximum.

If $\bar{u}$ is at point $A$, and the local maximum at point $B$ is the one desired (rather than the one at point $F$ ) some means must be employed to guarantee that (82) will produce a value of $u$ in the neighborhood of B. A value near $E$ will eventually converge to $F$. Thus (82) should be replaced by

$$
u=\bar{u}-\epsilon \frac{V^{\prime}(\bar{u})}{V^{\prime \prime}(\bar{u})}
$$

Then, (83) becomes

$$
V(u)-V(\bar{u})=\left(\frac{1}{2} \epsilon^{2}-\epsilon\right) \frac{V^{\prime}(\bar{u})^{2}}{V^{\prime \prime}(\bar{u})}
$$

Thus, an improvement may be guaranteed at every stage if (84) is used with proper choice of $\epsilon$, if the initial nominal lies somewhere to the left of point $D$.

If the nominal is to the right of point $E$, the algorithm will tend to point $F$.

Points between $\mathrm{C}$ and $\mathrm{E}$ are problematical because $\mathrm{V}^{\prime \prime}(\mathrm{u})$ is not negative-definite ${ }^{\dagger}$. In neighborhoods of $C$ and $E,(84)$ and (85) are not useable.

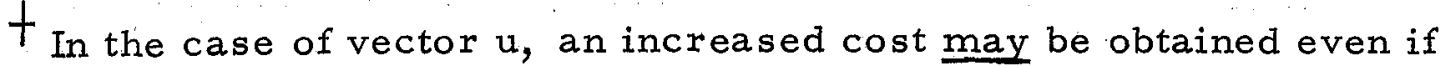
$V^{\prime \prime}(u)$ is non-negative-definite. In the scalar case this is not possible. 
This is not perfectly analogous to the algorithm for discretetime dynamic optimization algorithms, but some comparisons may be drawn. In the discrete dynamic case, u may be thought of as an $\mathrm{N}$-vector $(\mathrm{N}=100$ or 400$)$. Then $\mathrm{V}^{\prime}$ is a vector, $\mathrm{V}^{\prime \prime}$ is a matrix, and $\epsilon$ represents the step-size adjustment method. Figure 1 may be thought of as a graph of $\mathrm{V}$ as a function of $\mathrm{u}$ for constant, near optimal k. Point $B$ is the local maximum where $\theta_{1}=\theta_{2}=0$, and is shown in tables 1-3. Point $F$ is the maximum of table 4 .

Behavior due to a point analogous to $\mathrm{E}$ has been observed. Iteration began at point $\mathrm{A}$, for near optimal $\mathrm{k}$. The next value of $\mathrm{u}$ was to the right of point $\mathrm{D}$ (because $\mathrm{V}$ calculated at that point was greater than that of Table 1. In this case, $N=100, t_{N}=3.32$ ). In successive iterations, $V$ continued to increase, as did $\left|\theta_{1}\right|$ and $\left|\theta_{2}\right|$ because $u$ was chosen to maximize H. However, it was impossible to drive $\mathrm{a}^{\circ}$ (analogous to (8.5)) below a certain value. After a few iterations, $a^{\circ}$ began to increase. Finally, $a^{\circ}$ jumped from a typical value of less than $10^{-3}$ to more than 300 in one iteration. At that iteration, elements of $\mathrm{V}_{\mathrm{xx}}$ were of the order of 5000 . This situation corresponds to a point near $\mathrm{C}$ or $\mathrm{E}$ where $\mathrm{V}_{\text {uu }}$ is near singular (the singularity manifests itself in the large values of $\mathrm{V}_{\mathrm{xx}}$ and $\mathrm{a}^{\circ}$ ).

Thus, in order to guarantee proper convergence iterations must be restricted to the neighborhood of the relative minimum desired. In the present algorithm, the restrictions are accomplished by:

1) The choice of a 'sufficiently good' nominal.

2) Minimization of $\mathrm{H}\left(\mathrm{u}\right.$ ) (rather than the use of $\delta \mathrm{u}=-\mathrm{H}_{\mathrm{uu}}^{-1} \mathrm{H}$ as in [5], [6], [9] and [14]).

3) Test 2. 


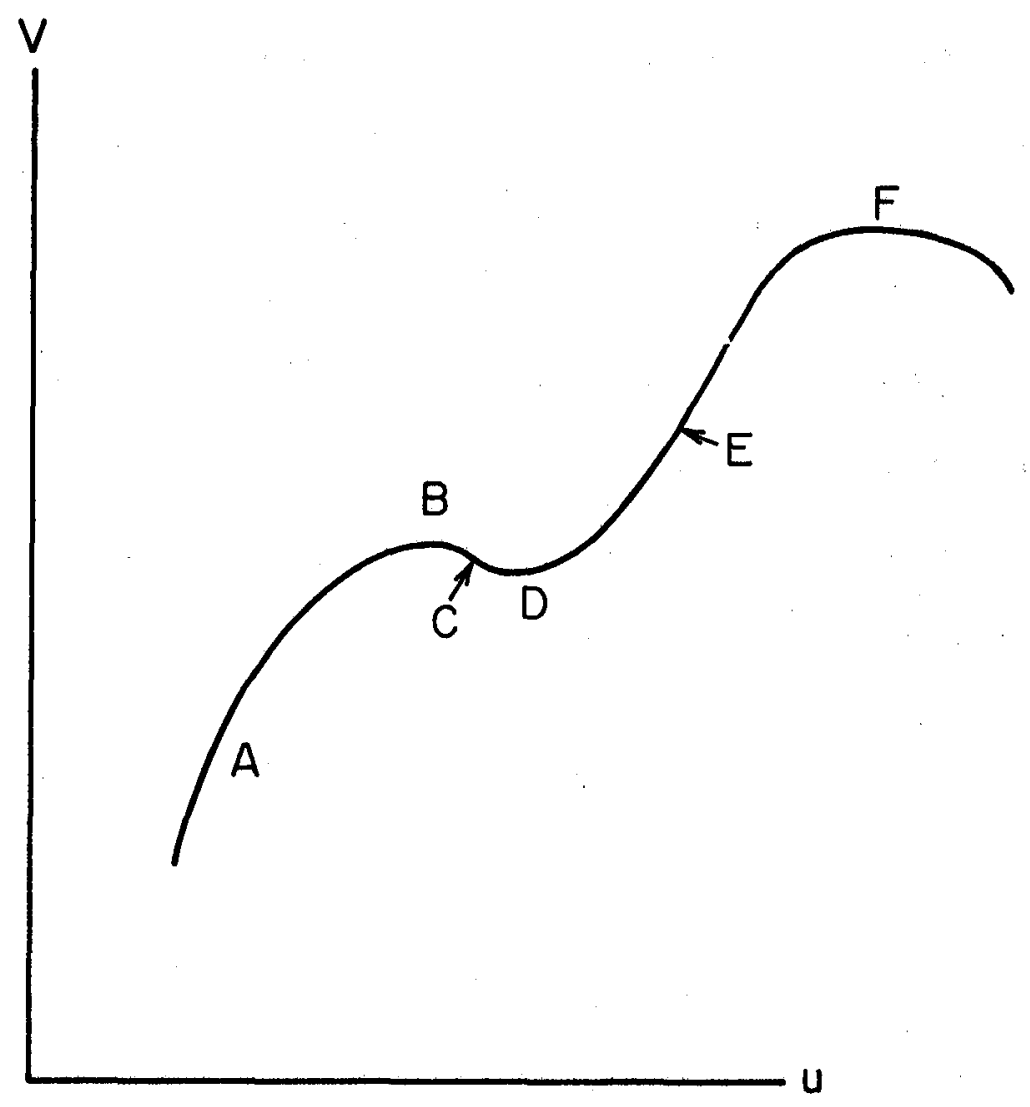

FIGURE 1 
V.4. Numerical Values of Tolerances

ETA, the criterion of optimality of $a^{\circ}$, was set to $10^{-2}$ and good results were obtained. Late in the iteration process, $a^{\circ}$ was always less and generally considerably less than this value, so that this constraint is rather ineffective. Earlier in the process, little is gained by requiring $\mathrm{a}^{\circ}$ to be extremely small, since that would require precise calculation of quantities which must change when $k$ is changed by $\delta k$, and which are non-critical.

Satisfactory results were obtained with CK and TOL, the tolerances of Test 1 and Test 2, respectively, set to $20 \%$ and $30 \%$. At less than $10 \%$, it became impossible to take steps sufficiently small in $\delta_{x_{i}}$ to satisfy Test 2. (This was found with $\mathrm{N}=100$. ) 
Table 1

\begin{tabular}{|c|c|c|c|c|c|c|c|}
\hline 100 & Time & Steps & $\longrightarrow$ & Final & Time & $=$ & 3.32 \\
\hline$t$ & $\mathrm{u}$ & $x_{1}$ & $x_{2}$ & $x_{3}$ & $\mathrm{~V}_{\mathrm{x}_{1}}$ & $\mathrm{~V}_{\mathbf{x}_{2}}$ & $\mathrm{~V}_{\mathbf{x}_{3}}$ \\
\hline 0 & .4430 & 1 & 0 & 1 & 1.8890 & .94316 & 2.0604 \\
\hline $\begin{array}{l}.166 \\
332\end{array}$ & .5188 & 1. 0008 & .0134 & 1,0201 & 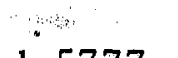 & & \\
\hline $\begin{array}{l}.332 \\
.498\end{array}$ & $\begin{array}{r}.6073 \\
.7097\end{array}$ & $\begin{array}{l}\text { 1. } 0044 \\
1.0121\end{array}$ & $\begin{array}{r}.0353 \\
0649\end{array}$ & $\begin{array}{r}1.0366 \\
10478\end{array}$ & 1. 5777 & .94982 & 1.4229 \\
\hline .664 & .8269 & $\begin{array}{l}1.0121 \\
1.0252\end{array}$ & .1011 & 1. 0520 & 1. 2963 & $\ldots 85152$ & \\
\hline .830 & .9592 & 1.0446 & .1419 & 1. 0479 & $2.0 / 0$ & & .82311 \\
\hline .996 & 1. 107 & 1.0711 & .1853 & 1.0349 & 1.0857 & .64554 & $\therefore 34816$ \\
\hline 1.162 & 1. 271 & 1.1047 & .2288 & 1.0129 & & & \\
\hline 1. 328 & 1. 460 & 1. 1455 & .2701 & .9823 & .96818 & .36222 & .055367 \\
\hline 1,894 & 1.730 & 1.1929 & .3071 & .9433 & & & \\
\hline 1.660 & 2. 886 & 1. 2459 & .3347 & .8924 & .93356 & .045050 & -.048480 \\
\hline 1.826 & 4.493 & 1. 3008 & .3157 & .8370 & & & \\
\hline 1.922 & 4.765 & 1. 3508 & .2786 & .8032 & .95639 & -.27469 & .0036793 \\
\hline 2.158 & 4.913 & 1. 3945 & .2390 & .7811 & & & \\
\hline 2.324 & 5.023 & 1. 4315 & 1991 & .7675 & 1. 0117 & -.58597 & .17505 \\
\hline 2.490 & 5.116 & 1. 4619 & .1600 & .7611 & & & \\
\hline $\begin{array}{l}2.656 \\
2.82 ?\end{array}$ & $\begin{array}{l}5.196 \\
5.269\end{array}$ & 1. 4860 & .1225 & .7609 & 1.1031 &.- .88384 & .44689 \\
\hline $\begin{array}{l}2.822 \\
2.988\end{array}$ & $\begin{array}{l}5.269 \\
5.335\end{array}$ & $\begin{array}{l}1.5039 \\
1.5162\end{array}$ & $\begin{array}{l}.0812 \\
.0546\end{array}$ & $\begin{array}{l}.1001 \\
.7762\end{array}$ & 1. 2105 & -1.1608 & 81108 \\
\hline 3. 154 & 5.398 & 1.5233 & .0254 & .7908 & & & \\
\hline 3.320 & & 1.5257 & .0000 & .8096 & 1.3356 & -1.4034 & 1. 2647 \\
\hline
\end{tabular}

Optimal V $=1.52572699$

$k_{1}=-1.40339248$

$\mathrm{k}_{2}=1.26501024$

$\theta_{1}=.75 \times 10^{-6}$

$\theta_{2}^{1}=.11 \times 10^{-6}$ 
Table 1

$t$

0

.332

.664

.996

1. 328

1. 660

1. 992

2. 324

2. 656

2. 988

3. 320

$t$<smiles>[Y][Y]=[V]</smiles>

0

.332

.664

.996

1. 328

1. 660

1. 992

2. 324

2. 656

2. 988

3. 320

t<smiles>[AlH2][AlH2]</smiles>

0

.332

.664

.996

1. 325

1. 660

1. 992

2. 324

2. 656

2. 988

3. 320
$\mathrm{V}_{\mathrm{x}_{1} \mathrm{x}_{1}}$

17.382

14. 024

10.142

6.5844

3. 8740

1. 6309

1. 0014

$\therefore 62113$

$10^{-3} \times .20185$

$-.33000$
$\mathrm{V}_{\mathrm{x}_{1} \mathrm{x}_{2}}$

5. 0412

5. 9021

5. 6729

4. 3821

2. 6629

.93085

.43781

.20735

.095264

.037090

0

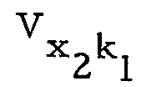

$$
\text { 1.1. } 763
$$

10. 134

7. 9954

5. 7024

3. 5734

1. 2686

.68877 .

.40892

.23166

.10279

0
1. 8948

3. 2408

3. 8989

3. 7125

2. 8099

1. 3089

1. 0293

.95075

.95901

.98678

1
$\mathrm{V}_{\mathrm{x}_{1}} \mathrm{x}_{3}$

25. 681

19. 323

12. 489

6. 8543

3. 2567

1. 2301

.83305

.68267

.51340

.29087

0

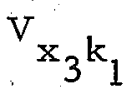

16. 457

13. 4.60

9. 8678

6. 3684

3. 5868

1. 5331

1. 0404

.84940

.61959

.33387

0

$\mathrm{V}_{\mathrm{k}_{1} \mathrm{k}_{2}}$

1. 1584

1. 0802

.97793

.83754

.63669

.28510

.19060

.10495

.053471

.061401

.020738

.020602
$V_{k_{2}} k_{2}$

.34241

.33200

.31744

.29576

.26168

.20310

.13001

.081612

.047200

.020546
${ }^{V} x_{2} x_{2}$

2. 7053

2. 5363

2. 6660

2. 3731

1. 5047

.40562

.17979

. 14331

.10731

.040093

0

$\mathrm{V}_{\mathrm{x}_{1} \mathrm{k}_{2}}$

2. 0921

1. 8111

1. 4737

1. 1258

.80242

.45836

.36011

.30271

.27821

.26806

.26537
$V_{x_{2} x_{3}}$

4. 1297

5.9353

5. 9028

4. 3792

2. 5309

1. 0976

.74599

.37114

.028400

$-.13086$

0

$\mathrm{V}_{\mathrm{x}_{2} \mathrm{k}_{2}}$

$\mathrm{V}_{\mathrm{x}_{3} \mathrm{k}_{2}}$

.47188

.60603

.62691

.51215

.28264

..023940

$-.16333$

-.19132

$-.15414$

-.084390

" 0

1
$\mathrm{V}_{\mathrm{x}_{3}} \mathrm{x}_{3}$

36. 371

26.238

15. 733

7. 5536

2. 7188

.34899

$-.72441$

$-1.1303$

$-1.0641$

$-.61585$

0

2. 6138

2. 0839

1. 5026

.976 .54

.59946

.38236

.56995

.74612

.88625

.97275 
Table 2

400 Time Steps $\cdots$ Final Time $=3.32$

\begin{tabular}{|c|c|c|c|c|c|c|c|}
\hline$t$ & $\mathbf{u}$ & $x_{1}$ & $x_{2}$ & $x_{3}$ & $\mathrm{~V}_{\mathrm{x}_{1}}$ & $\mathrm{~V}_{\mathrm{x}_{2}}$ & $\mathrm{~V}_{\mathbf{x}_{3}}$ \\
\hline $\begin{array}{l}0 \\
.166 \\
.332 \\
.498 \\
.664 \\
.830 \\
.996 \\
1.162 \\
1.328 \\
1.494 \\
1.660 \\
1.826 \\
1.992 \\
2.158 \\
2.324 \\
2.490 \\
2.656 \\
2.882 \\
2.988 \\
3.154 \\
3.320\end{array}$ & $\begin{array}{r}.4332 \\
.5072 \\
.5937 \\
.6936 \\
.8080 \\
.9371 \\
1.081 \\
1.241 \\
1.426 \\
1.683 \\
2.645 \\
4.437 \\
4.732 \\
4.885 \\
4.999 \\
5.093 \\
5.176 \\
5.250 \\
5.318 \\
5.382 \\
\end{array}$ & $\begin{array}{l}1 \\
1.0010 \\
1.0049 \\
1.0132 \\
1.0269 \\
1.0469 \\
1.0740 \\
1.1082 \\
1.1493 \\
1.1970 \\
1.2502 \\
1.3048 \\
1.3542 \\
1.3972 \\
1.4337 \\
1.4636 \\
1.4872 \\
1.5047 \\
1.5165 \\
1.5232 \\
1.5254\end{array}$ & $\begin{array}{l}0 \\
.0139 \\
.0361 \\
.0659 \\
.1020 \\
.1425 \\
.1855 \\
.2285 \\
.2693 \\
.3059 \\
.3335 \\
.3149 \\
.2780 \\
.2386 \\
.1988 \\
.1598 \\
.1224 \\
.0871 \\
.0546 \\
.0254 \\
.0000\end{array}$ & $\begin{array}{l}1 . \\
1.0200 \\
1.0362 \\
1.0470 \\
1.0507 \\
1.0464 \\
1.0332 \\
1.0114 \\
.9811 \\
.9428 \\
.8927 \\
.8375 \\
.8039 \\
. .7818 \\
.7682 \\
.7617 \\
.7613 \\
.7664 \\
.7765 \\
.7910 \\
.8097\end{array}$ & $\begin{array}{l}1.8803 \\
1.7254 \\
1.5729 \\
1.4273 \\
1.2942 \\
1.1790 \\
1.0857 \\
1.0160 \\
.96936 \\
.94344 \\
.93488 \\
.94036 \\
.95720 \\
.98321 \\
1.0168 \\
1.0569 \\
1.1029 \\
1.1541 \\
1.2102 \\
1.2709 \\
1.3356\end{array}$ & $\begin{array}{l}.93239 \\
.94700 \\
.93843 \\
.90323 \\
.83985 \\
.74911 \\
.63410 \\
.49963 \\
.35134 \\
.19473 \\
.034410 \\
-.12632 \\
-.28580 \\
-.44323 \\
-.59804 \\
-.74962 \\
-.89717 \\
-1.0396 \\
-1.1755 \\
-1.3029 \\
-1.4194\end{array}$ & $\begin{array}{l}2.0340 \\
1.7201 \\
1.4045 \\
1.0982 \\
.81261 \\
.55832 \\
.34405 \\
.17548 \\
.054908 \\
-.018536 \\
-.048200 \\
-.039267 \\
.0028600 \\
.074425 \\
.17286 \\
.29642 \\
.44398 \\
.61486 \\
.80871 \\
1.0253 \\
1.2646\end{array}$ \\
\hline
\end{tabular}

Optimal $\begin{aligned} V & =1.52537493 \\ k_{1} & =-1.41936325 \\ k_{2} & =1.26460750 \\ \theta_{1} & =-.33 \times 10^{-6} \\ \theta_{2} & =.37 \times 10^{-7}\end{aligned}$ 
Table 2

\begin{tabular}{|c|c|c|c|c|c|c|}
\hline$t$ & $v_{x_{1} x_{1}}$ & $x_{1} x_{2}$ & $v_{x_{1} x_{3}}$ & $V_{x_{2}} x_{2}$ & $v_{x_{2}} x_{3}$ & $\mathbf{v}_{\mathbf{x}_{3} \mathrm{x}_{3}}$ \\
\hline $\begin{array}{l}0 \\
.166 \\
.332 \\
.498 \\
.664 \\
.830 \\
.996 \\
1.162 \\
1.328 \\
1.494 \\
1.660 \\
1.826 \\
1.992 \\
2.158 \\
2.324 \\
2.490 \\
2.656 \\
2.822 \\
2.988 \\
3.154\end{array}$ & $\begin{array}{l}25.668 \\
23.004 \\
20.020 \\
16.875 \\
13.767 \\
10.887 \\
8.3712 \\
6.2725 \\
4.5578 \\
3.1074 \\
1.6814 \\
1.2733 \\
1.0094 \\
.80110 \\
.62423 \\
46343 \\
30967 \\
15707 \\
.0014626 \\
16020 \\
. .33005\end{array}$ & $\begin{array}{l}6.4714 \\
7.3407 \\
7.8261 \\
7.8472 \\
7.4030 \\
6.5734 \\
5.4944 \\
4.3143 \\
3.1493 \\
2.0442 \\
.96700 \\
.66432 \\
44680 \\
.30614 \\
21096 \\
.14436 \\
.096961 \\
.062838 \\
.037691 \\
017914 \\
0 .\end{array}$ & $\begin{array}{l}36.811 \\
32.083 \\
26.940 \\
21.711 \\
16.748 \\
12.357 \\
8.7324 \\
5.9299 \\
3.8821 \\
2.4311 \\
1.2982 \\
.95119 \\
.85425 \\
.77199 \\
69358 \\
.61088 \\
51862 \\
.41337 \\
29278 \\
15530 \\
0\end{array}$ & $\begin{array}{l}2.9982 \\
3.0133 \\
3.1994 \\
3.4165 \\
3.5261 \\
3.4270 \\
3.0840 \\
2.5329 \\
1.8550 \\
1.1295 \\
.42449 \\
26681 \\
17135 \\
14147 \\
13159 \\
12113 \\
.10202 \\
.073672 \\
040783 \\
012180 \\
0\end{array}$ & $\begin{array}{l}6.1215 \\
7.5962 \\
8.4188 \\
8.5199 \\
7.9529 \\
6.8843 \\
5.5498 \\
4.1859 \\
2.9672 \\
1.9686 \\
1.1349 \\
.91097 \\
.75975 \\
.58102 \\
.39144 \\
.20821 \\
.050278 \\
-.063771 \\
-.11762 \\
-.098794 \\
0\end{array}$ & $\begin{array}{l}51.271 \\
43.827 \\
35.869 \\
27.975 \\
20.696 \\
14.457 \\
9.4885 \\
5.8081 \\
3.2561 \\
1.5713 \\
.39922 \\
-31000 \\
-68761 \\
-.95063 \\
-1.0988 \\
-1.1304 \\
-1.0504 \\
-.87208 \\
-.61715 \\
-31479 \\
0\end{array}$ \\
\hline
\end{tabular}


Table 2

$t$

0

.166

.332

.498

.664

.830

.996

1. 162

t.o 328

1. 494

1. 660

1. 826

1.992

2. 158

2. 324

2. 490

2. 656

2. 822

2. 988

3. 154

3. 320

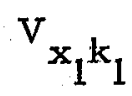

16. 324

15. 077

13. 635

12. 044

10. 369

8. 6893

7. 0750

5. 5754

4.1963

2. 8623

1. 3086

.90514

.69021

.53029

.40854

.31148

.23115

.16260

.10257

.048862 $v_{x_{2}} k_{1}$

2. 7072

3. 6576

4. 3937

4. 8705

5. 0587

4. 9548

4. 5854

4. 0023

3. 2638

2. 3949

1. 3438

1. 1591

1. 0388

.97807

.95379

.95055

.95886

.97224

.98591

.99615
$\mathrm{V}_{\mathrm{x}_{3} \mathrm{k}_{1}}$

22. 568

20.360

17. 898

15. 288

12. 652

10. 119

7.8073

5. 8054

4. 1519

2. 8156

1. 5850

1. 1539

1. 0480

.94985

.84945

.74012

.61829

.48265

.33340

.17185

0
$\mathrm{V}_{\mathrm{x}_{2} \mathrm{k}_{1}}$

2. 9965

2. 7752

2. 5261

2. 2562

1. 9757

1. 6962

1. 4283

1. 1785

.94615

.71731

.47315

.42623

.36451

.32738

.30386

.28843

.27827

.27178

.26792

.26598

.26540

$$
\mathrm{V}_{\mathrm{x}_{2} \mathrm{k}_{2}}
$$

.62088

.74548

.83261

.87461

.86650

.80765

.70248

.55971

.38932

.19653

$-.0064651$

$-.084704$

-. 15287

-. 18133

$-.18620$

$-.17506$

$-.15239$

$-.12132$

$-.084297$

$-.043302$

0 $v_{x_{3}} k_{2}$

3. 8397

3. 4393

3. 0041

2. 5524

2. 1047

1. 6828

1. 3065

.99064

.74192

.55547

.41096

.48855

.57766

.66436

.74675

.82111

.88454

.93504

.97138

.99302 
Table 2

$t$

0

.166

.332

.498

.664

.830

.996

1. 162

1. 328

1. 494

1. 660

1. 826

1. 992

2. 158

2. 324

2. 490

2. 656

2. 822

2. 988

3. 154

3. 320
$\mathrm{V}_{\mathrm{k}_{1} \mathrm{k}_{1}}$

8. 8160

8. 3022

7. 7593

7. 1776

6. 5494

5. 8690

5. 1333

4. 3363

3. 4536

2. 3868

.77092

.41196

.29083

.20165

.13913

.094332

.061797

.038102

.020935

.0086435

0
$\mathrm{V}_{\mathrm{k}_{1} \mathrm{k}_{2}}$

1. 6610

1. 5836

1. 5002

1. 4088

1. 3075

1. 1947

1. 0687

.92737

.76489

.56341

.29390

.26081

.19216

.14280

.10584

.076993

.054015

.035595

.020859

.0091655
$\mathrm{V}_{2} \mathrm{k}_{2} \mathrm{k}_{2}$

43881

42715

41432

.39995

.38363

. 36492

.34336

.31828

.28837

.25032

.20465

.17086

.13187

.10454

.082684

.064096

.047855

.033524

.020865

.0097293 $\beta_{1}$

1,6117

1. 6380

1. 6858

1.7499

1. 8270

1. 9211

2. 0551

2. 3043

2. 9216

5. 2092

35.639

$-9.1970$

$-14.033$

$-9.4342$

$-4.7097$

1. 6346

12. 727

37,541

114. 52

593.49

$\infty$ $\beta_{2}$

.92861

.92017

.94436

1. 0069

1. 1172

1. 2932

1. 5759

2.0820

3. 2442

7. 7912

100,Q4

$-21.079$

$-86.862$

$-98.806$

$-118.50$

$-153.98$

$-221.03$

$-366.27$

$-781.63$

$-3112.0$

$\infty$ $\beta_{3}$

1. 2998

1. 3739

1. 4882

1. 6473

1. 8637

2. 1662

2. 6231

3. 4117

5. 0950

10.505

71.209

$-23.071$

$-25.854$

$-14.020$

$-\quad 13581$

21. 075

60.962

115.3. 11

442.30

2247.1

$\infty$ 
Table 3

400 Time Steps - Final Time $=3.3194$

\begin{tabular}{|c|c|c|c|c|c|c|c|}
\hline$t$ & $\mathrm{u}$ & $x_{1}$ & $x_{2}$ & $x_{3}$ & $\mathrm{~V}_{\mathrm{x}_{1}}$ & $\mathrm{~V}_{\mathrm{x}_{2}}$ & $\mathrm{~V}_{\mathbf{x}_{3}}$ \\
\hline 0 & .4333 & 1 & 0 & 1 & 1.8800 & -.93244 & 2. 0334 \\
\hline .166 & .5074 & 1.0010 & .0139 & 1. 0199 & 1.7252 & .94699 & 1. 7196 \\
\hline .332 & .5938 & 1.0049 & .0361 & 1. 0362 & 1.5727 & 93838 & 1. 4041 \\
\hline 498 & 6937 & 1. 0131 & .0658 & 1. 0470 & 1. 4272 & 90314 & 1.0979 \\
\hline 664 & 8080 & 1.0268 & .1019 & 1.0507 & 1. 2941 & .83974 & .81232 \\
\hline .830 & .9372 & 1.0469 & .1425 & 1. 0464 & 1.1790 & .74899 & 55811 \\
\hline .996 & 1.081 & 1.0739 & .1854 & 1. 0332 & 1. 0856 & .63399 & 34391 \\
\hline 1.162 & 1. 242 & 1.1081 & .2284 & 1. 0114 & 1. 0160 & .49953 & .17540 \\
\hline 1. 328 & 1. 426 & 1,1493 & .2692 & .9812 & .96934 & 35127 & .054860 \\
\hline 1. 494 & 1.683 & 1.1969 & .3059 & 9429 & .94343 & .19468 & -.018561 \\
\hline 1.660 & 2.645 & 1. 2501 & .3334 &. .8927 & .93487 & .034386 & -.048213 \\
\hline 1.826 & 4.437 & 1.3046 & .3148 & .8375 & .94035 & -.12631 & -.039278 \\
\hline 1.992 & 4.732 & 1. 3540 & .2779 & .8040 & .95720 & -.28576 & 002844 \\
\hline 2.158 & 4.885 & 1.3971 & .2386 & .7819 & .98321 & $=44317$ & 074400 \\
\hline 2. 324 & 4.999 & 1. 4335 & .1988 & .7682 & 1. 0168 & $\because 59796$ & 17282 \\
\hline 2.490 & 5.093 & 1. 4634 & .1598 & .7617 & 1.0569 & -74951 & 29636 \\
\hline 2.656 & 5.176 & 1. 4870 & .1223 & .7614 & 1.1029 & $\therefore 89703$ & 44390 \\
\hline 2.821 & 5.250 & 1.5045 & .0871 & .7665 & 1. 1541 & 40394 & 61476 \\
\hline & 5.318 & 1. 5163 & .0546 & .7765 & 1. 2103 & -1.1753 & 80858 \\
\hline & 5.382 & 1. 5230 & .0254 & .79 & 1. 2709 & -1.3026 & 1.0252 \\
\hline & - & 1. 5252 & .0000 & .8097 & 1. 3357 & -1.4191 & 1. 2644 \\
\hline
\end{tabular}

Optimal $\mathrm{V}=1.52516085$

$k_{1}=-1.41910912$

$k_{2}=1.26441935$

$\theta_{1}=-10 \times 10^{-5}$
$\theta_{2}=-.26 \times 10^{-6}$ 
Table 3

$t$<smiles>[Y][Y]</smiles>

0

.166

.332

.498

.664

.830

.996

1. 162

1. 328

1. 494

1. 660

1. 826

1. 992

2. 158

2. 324

2. 490

2. 656

2. 821

2. 987

3. 153

3. 319

\begin{abstract}
25. 654
\end{abstract}
22. 991

20.009

16.866

13. 760

10. 882

8. 3684

6. 2708

4. 5568

3. 1069

1. 6813

1. 2735

1. 0096

.80125

.62436

.46354

.30975

.15712

.0014710

-. 16023

$-.33013$
$\mathrm{V}_{\mathrm{x}_{1} \mathrm{x}_{2}}$

6. 4705

7. 3385

7. 8231

7. 8437

7. 3995

6. 5703

5. 4918

4. 3124

3. 1480

2. 0433

.96672

.66426

.44678

.30613

.21096

. 14436

.096968

.062844

.037694

0
$V_{x_{1} x_{3}}$

36. 789

32.064

26. 924

21.698

16.739

12. 351

8. 7288

5. 9278

3. 8811

2. 4306

1. 2981

.95129

.85434

.77206

.69364

.61091

.51865

.41338

.29279

.15530
$\mathrm{V}_{2} \mathrm{x}_{2}$

2. 9974

3. 0124

3. 1981

3. 4148

3. 5240

3. 4247

3. 0819

2. 5311

1. 8536

1. 1286

.42418

.26668

.17128

.14142

.13156

.12111

.10201

.073662

.040778 $\mathrm{v}_{\mathrm{x}_{2}} \mathrm{x}_{3}$

6. 1205

7. 5935

8. 4148

8. 5154

7. 9484

6. 8805

5. 5469

4. 1839

2. 9660

1. 9680

1. 1346

.91091

.75970

.58099

.39141

. 20820

.050286

.063752

$-.11760$

.098777 $v x_{3} x_{3}$

51. 239

43. 799

35.846

27.958

20.684

14. 449

9. 4838

5. 8055

3. 2549

1. 5708 .39905

$-.30994$

$-.68749$

. .95047

$-1.0986$

$-1.1302$

$-1.0502$

$-.87192$

$-.61704$

$-.31473$ 
Table 3

t

$\begin{array}{lll}0 & 16.317 & 2.7075 \\ .166 & 15.071 & 3.6572 \\ 332 & 13.630 & 4.3926 \\ .498 & 12.039 & 4.8689 \\ .664 & 10.366 & 5.0568 \\ .830 & 8.6864 & 4.9528 \\ .996 & 7.0729 & 4.5835 \\ 1.162 & 5.5739 & 4.0006 \\ 1.328 & 4.1953 & 3.2625 \\ 1.494 & 2.8616 & 2.3940 \\ 1.660 & 1.3084 & 1.3435 \\ 1.826 & .90522 & 1.1590 \\ 1.992 & .69030 & 1.0388 \\ 2.158 & .53037 & .97804 \\ 2.324 & .40861 & .95377 \\ 2.490 & .31154 & .95054 \\ 2.656 & .24120 & .95886 \\ 2.821 & .16264 & .97224 \\ 2.987 & .10259 & .98590 \\ 3.153 & .048875 & .99615 \\ 3.319 & .00 & 1\end{array}$<smiles>[Y19][As]</smiles>

22. 557

20. 351

17. 890

15. 281

12. 647

10. 115

7. 8046

5. 8036

4. 1508

2. 8151

1. 5848

1. 1540

1. 0481

.94994

84953

74018

.61834

.48268

.33342

.17186

0 $\mathrm{v}_{\mathrm{x}_{1} \mathrm{k}_{2}}$

2. 9951

2. 7740

2. 5250

2. 2552

1. 9749

1. 6956

1. 4279

1. 1782

.94593

.71717

47314

42626

36455

32743

30391

28848

27832

.27183

.26798

.26604

.26546 $\mathrm{v}_{\mathrm{x}_{2} \mathrm{k}_{2}}$

$\mathrm{V}_{\mathrm{x}_{3} \mathrm{k}_{2}}$

. .62084

3. 8376

.74530

3. 4374

.83230

3. 0025

.87420

2. 5510

.86604

2. 1036

.80718

.70203

1. 6819

.55932

1. 3059

38900

.19629

$-.0065624$

.99027

.74171

.55536

-. 084763

.41096

$-.15291$

-. 18136

$-.18622$

$-.17508$

-. 15240

-. 12133

-. 084302

-. 043304

0
.48859

.57770

.66440

.74678

.82113

.88456

.93505

.97139

.99303 
Table 3

$\begin{array}{lllllll}\mathrm{t} & \mathrm{V} \mathrm{k}_{1} \mathrm{k}_{1} & \mathrm{~V}_{\mathrm{k}_{1} \mathrm{k}_{2}} & \mathrm{~V}_{\mathrm{k}_{2} \mathrm{k}_{2}} & \beta_{1} & \beta_{2} & \beta_{3} \\ 0 & 8.8130 & 1.6604 & .43870 & 1.6118 & .92857 & 1.3000 \\ .166 & 8.2995 & 1.5831 & .42704 & 1.6381 & .92017 & 1.3742 \\ .332 & 7.7568 & 1.4997 & .41422 & 1.6860 & .94440 & 1.4886 \\ .498 & 7.1754 & 1.4083 & .39986 & 1.7501 & 1.0070 & 1.6478 \\ .664 & 6.5474 & 1.3071 & .38354 & 1.8273 & 1.1174 & 1.8643 \\ .830 & 5.8673 & 1.1943 & .36485 & 1.9215 & 1.2934 & 2.1669 \\ .996 & 5.1318 & 1.0684 & .34330 & 2.0557 & 1.5762 & 2.6241 \\ 1.162 & 4.3350 & .92709 & .31823 & 2.3051 & 2.0825 & 3.4131 \\ 1.328 & 3.4525 & .76466 & .28833 & 2.9229 & 3.2455 & 5.0974 \\ 1.494 & 2.3859 & .56322 & .25029 & 5.2124 & 7.7957 & 10.511 \\ 1.660 & .77050 & .29385 & .20465 & 35.663 & 100.10 & 71.248 \\ 1.826 & .41195 & .26081 & .17086 & -9.1921 & -21.042 & -23.063 \\ 1.992 & .29084 & .19217 & .13187 & -14.038 & -86.876 & -25.861 \\ 2.158 & .20165 & .14280 & .10454 & -9.4381 & -98.827 & -14.025 \\ 2.324 & .13913 & .10584 & .082681 & -4.7120 & -118.53 & -.13744 \\ 2.490 & .094332 & .076991 & .064092 & 1.6350 & -154.02 & 21.079 \\ 2.656 & .061797 & .054013 & .047852 & 12.732 & -221.08 & 60.976 \\ 2.821 & .038101 & .035593 & .033521 & 37.558 & -366.35 & 153.15 \\ 2.987 & .020934 & .020858 & .020863 & 114.57 & -781.82 & 442.42 \\ 3.153 & .0086431 & .0091649 & .0097283 & 593.80 & -3112.9 & 2247.8\end{array}$


Table 4

\begin{tabular}{|c|c|c|c|c|c|c|c|}
\hline & 100 & Time & $s$ & Final & Time & $=3.32$ & \\
\hline$t$ & $\mathrm{u}$ & $x_{1}$ & $\mathrm{x}_{2}$ & $x_{3}$ & $\mathrm{v}_{\mathrm{x}_{1}}$ & $\mathrm{~V}_{\mathrm{x}_{2}}$ & $\mathrm{v}_{\mathrm{x}_{3}}$ \\
\hline 0 & 1. 147 & 1. & 0 & 1 & 1.5399 & 3.8503 & 1. 5607 \\
\hline .166 & 1. 458 & 1.0015 & .0233 & 1. 0055 & & & \\
\hline .332 & 1. 802 & 1.0071 & 0489 & .9993 & .065221 & 3. 4865 & -1.1789 \\
\hline .498 & 2.172 & 1.0167 & 0706 & .9812 & & & \\
\hline .664 & 2. 423 & 1.0294 & .0826 & .9529 & -1.2528 & 2.6667 & -3.5848 \\
\hline .830 & 2.674 & 1.0433 & .0833 & .9200 & & & \\
\hline .996 & 2. 819 & 1.0565 & .0722 & .8850 & -2.0365 & 1.7928 & -5.1481 \\
\hline 1.162 & 2.917 & 1.0672 & .0508 & .8525 & & & \\
\hline 1.328 & 2. 990 & 1.0738 & .0203 & .8221 & -2.4128 & $I_{\infty} 0467$ & -5.9689 \\
\hline 1.494 & 3.048 & 1.0747 & -.0184 & .7956 & & & \\
\hline 1.660 & 3.098 & $1.0686^{\circ}$ & -0650 & .7737 & -2.6385 & .39794 & -6.2592 \\
\hline 1.826 & 3.143 & 1.0543 & -.1194 & 7572 & & & \\
\hline 1.992 & 3.186 & 1.0305 & -.1818 & .7472 & -2.7998 & -.14885 & -6.0700 \\
\hline 2.158 & 3.229 & .9957 & -.2532 & .7452 & & & \\
\hline 2. 324 & 3.275 & .9484 & -.3349 & .7536 & -2.9268 & -.59906 & -5.3983 \\
\hline 2.490 & 3.331 & .88 & -.4294 & .7765 & & & \\
\hline 2.656 & 3.406 & .8083 & -.5404 & .8211 & -2.9898 & -.97790 & -4.1832 \\
\hline 2. 82 & 3.528 & .7100 & -.6738 & .9021 & & & \\
\hline 988 & 3.778 & .5878 & -.8374 & 1.0534 & -2.6070 & -1.3288 & -2.2274 \\
\hline 15 & 4.473 & .4361 & -1.0316 & 1. 3731 & & & \\
\hline & & 2559 & 0621 & 2.2275 & 5.8682 & -1.3996 & 1.2600 \\
\hline
\end{tabular}

$\mathrm{V} \quad 2.05800182$

$k_{1}=-1.3996310$

$k_{2}=: 1.2600310$

$\theta_{1}=-1.0620840$

$\theta_{2}=0.25048833$ 
Table 4

t<smiles>[Y][Y][3H]</smiles>

$-24.414$

$-36.687$

$-53.131$

$-61.956$

$-61.104$

$-51.538$

$-33.480$

$-8.6663$

18. 256

39. 504

$-28.541$

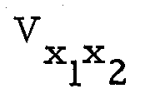

$-2.0022$

$-17.856$

$-41.090$

$-.58 .592$

$-65.932$

$-62.151$

$-48.075$

$-27.949$

$-9.1917$

1. 1026

0
$V_{x_{1} x_{3}}$

$-37.216$

$-47.037$

$-54.533$

$-47.827$

$-27.929$

$-1.0216$

24.962

40.562

39.034

21. 040

0
$\mathrm{V}_{\mathrm{x}_{2}} \mathrm{x}_{2}$

20.959

7. 6941

$-13.587$

$-35.420$

$-49.859$

$-52.137$

$-41.927$

$-24.679$

$-9.0632$

$-.90940$

0
$\mathrm{V}_{\mathrm{x} \mathrm{x}_{3}}$

$-7.9807$

$-30.388$

$-51.207$

$-58.469$

$-48.845$

$-27.091$

$-3.0412$

12.112

13. 118

4. 8651

0
$\mathrm{V}_{\mathrm{x}_{3}} \mathrm{x}_{3}$

$-43.107$

$-45.222$

$-37.624$

$-12,146$

$-21.572$

48. 156

55. 434

41.086

16. 361

$-.70196$

0 
Table 4

$\begin{array}{llllll}\mathrm{V}_{\mathrm{x}_{1} \mathrm{k}_{1}} & \mathrm{~V}_{\mathrm{x}_{2} \mathrm{k}_{1}} & \mathrm{~V}_{\mathrm{x}_{3} \mathrm{k}_{1}} & \mathrm{~V}_{\mathrm{x}_{1} \mathrm{k}_{2}} & \mathrm{~V}_{\mathrm{x}_{2} \mathrm{k}_{2}} & \mathrm{~V}_{\mathrm{x}_{3} \mathrm{k}_{2}} \\ -.63978 & -1.8778 & -.73634 & .28581 & 1.6463 & .11388 \\ -.058647 & -1.8077 & .47180 & -.055295 & 1.3166 & -1.2612 \\ .22644 & -1.6848 & 1.3543 & -1.4659 & .65052 & -2.5802 \\ .20785 & -1.6316 & 1.9042 & -2.2572 & -.18701 & -3.5000 \\ .055229 & -1.6358 & 2.3214 & -2.9432 & -1.0756 & -3.9021 \\ -.11039 & -1.6351 & 2.7404 & -3.4968 & -1.8585 & -3.6752 \\ -.14652 & -1.5086 & 3.2172 & -3.7835 & -2.3211 & -2.7753 \\ .10870 & -1.1360 & 3.6343 & -3.6113 & -2.2884 & -1.3815 \\ .77093 & -.50338 & 3.6627 & -2.8160 & -1.7731 & .081057 . \\ 1.8439 & 2.9461 & 2.8218 & -1.1017 & -.96980 & 1.1273 \\ 0 & 1 & 0 & 3.8635 & 0 & 1\end{array}$


Table 4

$t$

0

.332

.664

.996

1. 328

1. 660

1. 992

2. 324

2. 656

2. 988

3. 320 $\mathrm{v}_{\mathrm{k}_{1} \mathrm{k}_{1}}$

.17173

.17168

.17005

.16428

.15402

.13853

.11778

.093937

.070021

.043426

0

$$
\mathrm{V}_{\mathrm{k}_{1} \mathrm{k}_{2}}
$$

.22694

.22658

.22270

.21388

.19916

.17695

.14737

.11390

.081529

.048409

0
$\mathrm{V}_{\mathrm{k}_{2} \mathrm{k}_{2}}$

.30997

.30454

.29477

.28121

.26008

.22827

.18608

.13910

.095279

.053988

0 
VI. Conclusion

A new discrete algorithm has been derived which is analogous to the continuous algorithm of [1] and [2]. Extensions to the latter (Test 1 and Test 2) have been developed to ensure that the new iterate is in the neighborhood of the current nominal.

The algorithm has been used to solve a non-linear, optimal orbit transfer problem. This problem has been attempted, and solved, in various forms, by a number of investigators using different computational methods.

The results obtained in this paper agree most closely with those of [12]. 
[1] D. H. Jacobson, Differential Dynamic Programming Methods for Determining Optimal Control of Non-Linear Systems, Ph。 D。 Thes is, Imperial College of Science and Technology, University of London, October, 1967.

[2] D. H. Jacobson, New Second Order and First Order Algorithms for Determining Optimal Control: A Differential Dynamic Programming Approach, Technical R eport. 551, Division of Engineering and Applied Physics, Harvard University, February, 1968 also J. Opt. Theory Applns。2, No, 6, 1968 to appear.

[3] $R$ 。 Bellman and $R$ 。 Kalaba, Dynamic Programming and Modern Control Theory, Academic Press, New York, 1965.

[4] S. R。 McReynolds, A Successive Sweep Method for Solving Optimal Control Problems, $\mathrm{Ph}$ 。 $\mathrm{D}$ 。Thesis, Harvard University, Cambridge, 1965 .

[5] S. R. McR eynolds, The Successive Sweep Method and Dynamic Programming, Journal of Mathematical A nalysis and Applications, Vol. 19, No。3, pp 565-598 September, 1967.

[6] S. R. McR eynolds and A, E. Bryson, Jr, A Successive Sweep Method for Solving Optimal Programming Problems, J.A.C.C., $6,1965,551$ 。

[7] H. J. Kelley, R。 E. Kopp, H. G. Moyer, A Trajectory Optimization Technique Based Upon the Theory of the Second Variation, AIAA Astrodynamics Conference, New Haven, Conn, August, 1963.

[8] H. J. Kelley, Method of Gradients, Optimization Techniques, Ed. G. Leitman, Academic Press, 1961, Ch 6。

[9] D. Q. Mayne, A Second Order Gradient Method of Optimizing Non-Linear Discrete-Time Systems, Int, J. Control, 3, 1966, 85.

[10] S. K. Mitter, Successive A pproximation Methods for the Solution of Optimal Control Problems, Automatica, Vol。3, 1966 pp 135-149.

[11] T. E. Bullock and G. F. Franklin, A Second-Order Feedback Method for Optimal Control Computations, IEEE Trans/on A uto. Control。AC-12, 1967, p 666 。

[12] B. D. Tapley and J.M. Lewallen, Comparison of Several Numerical Optimization Methods, Journal of Optimization Theory and A pplications, Vol. 1, No. 1, July 1967.

[13] A. E. Bryson and $Y, C$. Ho, Optimization, Estimation and Control, Blaisdell Publishing Co, Waltham, Mass, 1968 to appear, Chs, 5-7.

[14] S. E. Dreyfus, The Numerical Solution of Non-Linear Optimal Control Problems, in Numerical Solutions of Non-Linear Differential Equations, D. Greenspan, Editor, John Wiley \& Sons, 1966, pp 97-113. 


\section{Appendix A}

Continuous Results from Jacobson

The following is a statement and solution of the continuous-time optimal control problem solved in [1]. The notation has been modified to conform to that of this paper. Thus some expression involving derivatives have been transposed, and $\sim$ has been placed over certain symbols to coincide with section III. 1 , above.

Problem: given that

$A-1$

$$
\dot{x}=\tilde{f}(x, u, t) ; \quad x\left(t_{0}\right)=x_{0}
$$

Find $u(t), t \in\left[t_{0}, t_{f}\right]$ to minimize

$A-2$

$$
\hat{V}\left(x_{0}, t_{0}\right)=\int_{t_{0}}^{t_{f}} \widetilde{L}(x, u, t) d t+F\left(x\left(t_{f}\right)\right)
$$

while satisfying

A-3

$$
\theta\left(x\left(t_{f}\right)\right)=0
$$

The constraints $(A-3)$ are adjoined to the cost functional $(A-2)$ :

$\mathrm{A}-4$

$$
V\left(x_{0}, t_{0}\right)=\hat{V}+k^{T} \theta\left(x\left(t_{f}\right)\right)
$$

The solution is:

$$
\begin{array}{ll}
A-5 & \beta_{1}=-\tilde{H}_{u u}^{-1}\left(\tilde{H}_{u x}+\tilde{f}_{u}^{T} v_{x x}\right) \\
A-6 & \beta_{2}=-\tilde{H}_{u u_{u}^{-1}}^{-1} \tilde{f}_{x k}^{T} \\
A-7 & -\dot{a}=\tilde{H}-\tilde{\widetilde{H}} \\
A-8 & -\dot{V}_{x}=\tilde{H}_{x}+(\tilde{f}-\overline{\tilde{f}}) V_{x x} \\
A-9 & -\dot{V}_{k}=(\tilde{f}-\tilde{\tilde{f}}) V_{x k}
\end{array}
$$


A- 10

$$
-\dot{\mathrm{V}}_{\mathrm{xk}}=\left(\tilde{f}_{\mathrm{x}}^{\mathrm{T}}+\beta_{1}^{\mathrm{T}} \tilde{f}_{\mathrm{u}}^{\mathrm{T}}\right) \mathrm{V}_{\mathrm{xk}}
$$

A- 11

$$
-\dot{V}_{\mathrm{kk}}=-\mathrm{V}_{\mathrm{xk}}^{\mathrm{T}} \tilde{\tilde{f}} \tilde{\mathrm{H}}_{\mathrm{uu}}^{-1} \tilde{f}_{\mathrm{u}}^{\mathrm{T}} \mathrm{V}_{\mathrm{xk}}
$$

$A-12$

$$
-\dot{V}_{x x}=\tilde{H}_{x x}+\tilde{f}_{x}^{T} V_{x x}+V_{x x} \tilde{f}_{x}-\left(\tilde{H}_{u x}+f_{u}^{T} v_{x x}\right)^{T} \tilde{H}_{u u}^{-1}\left(H_{u x}+f_{u}^{T} v_{x x}\right)
$$

where $\tilde{H}=\widetilde{L}+V_{x} \tilde{f}$, and derivatives of $H$ are taken with $V_{x}$ constant, i.e.

$$
\widetilde{H}_{x}=\widetilde{L}_{x}+V_{x} \tilde{f}_{x}
$$

The boundary conditions of $(A-7)$ through $(A-12)$ are the same as equations (33)-(38) above. 


\section{Appendix B}

The Computer Program

Implementation of the algorithm on the problem described in section three required the use of a computer. A program has been written for the IBM 7094 in FORTRAN IV, which consists of several subprograms.

1. MAIN

This program is described in Flow Chart II in general outline. This program coordinates the algorithm. It starts by setting initial quantities, and quantities which do not change throughout the computation. Included are input numbers, constant elements of $\tilde{f}_{x}$ and $\tilde{f}_{x x}$, and constant boundary conditions.

The routine FORINT is called, which integrates the state equations (1). On the first iteration, the initial nominal control history is used. Subsequently, $u_{i}$ is calculated in FORINT. The performance index and terminal constraints are evaluated.

The calling of FORINT is part of the "step-size adjustment", as described in [1] and [2] and Flow Chart I.

Once a suitable trajectory is calculated, it is printed out and BAKINT is called to integrate the equations for $a^{i}, v_{x}^{i}$, and $v_{x x}^{i}$. If the absolute values of $a^{\circ}$ and the terminal constraints are less than ETA, ETA1, and ETA2, respectively (which are input quantities), iteration ceases. The routine BETA is called, which calculates the optimal feedback vector $\beta$ such that on a path slightly perturbed from the optimal, $\delta_{u}=\beta^{T} \delta_{x}$.

If $\mathrm{a}^{\mathrm{O}}$ is not smaller than ETA in absolute value, the program transfers to the forward integrator to improve the nominal trajectory. 
When the trajectory has been optimized for a given value of $k$, i.e., when $\mathrm{a}^{\circ}$ is driven to less than ETA, the routine DKCALC is called, which integrates the $\mathrm{V}_{\mathrm{kk}}^{\mathrm{i}}$ and $\mathrm{V}_{\mathrm{xk}}^{\mathrm{i}}$ equations, and calculates $\delta \mathrm{k}$ according to (59). The value of $\epsilon$ is originally 1 , , but if each component of $\theta$ is not decreased (by the introduction of $\delta \mathrm{k}$ ) in absolute value, and if the change in performance index is not within a tolerance (an input quantity) of the value predicted by (60) (i.e., if Test 1 is failed), then $\epsilon$ is reduced by half and the forward integrator is called again to calculate $\theta$ and $V$. When the criteria are satisfied, $\bar{k}$ is replaced by $\bar{k}+\delta \mathrm{k}$ and the program transfers to BAKINT.

\section{FORINT}

This routine integrates (1) forward. It calculates $u_{i}$ by maximizing

$$
H\left(\bar{x}_{i}+\delta_{x_{i}}, u_{i}, k+\delta k, t_{i}\right)=V_{x}^{i+1}\left(\bar{x}_{i+1}+\delta_{x_{i+1}}, k+\delta k\right) f\left(\bar{x}_{i}+\delta_{x_{i}}, u_{i}, t_{i}\right),
$$

which is equivalent to maximizing

$$
\begin{aligned}
& E=C \sin u_{i}+D \cos u_{i}, \quad \text { where } \\
& C=v_{x_{2}}^{i+1}\left(\bar{x}_{i+1}+\delta x_{i+1}, \bar{k}+\delta k\right) \\
& D=v_{x_{3}}^{i+1}\left(\bar{x}_{i+1}+\delta x_{i+1}, \bar{k}+\delta k\right)
\end{aligned}
$$

$\mathrm{C}$ and $\mathrm{D}$ are calculated by expanding $\mathrm{V}_{\mathrm{x}}^{\mathrm{i}+1}$ in $\delta_{\mathrm{x}_{\mathrm{i}+1}}$ and $\delta \mathrm{k}$. However, $\delta_{x_{i}}$ is used in place of $\delta_{x_{i+1}}$. See section IV. 1 .

At the maximum of $E$,

$$
u_{i}=\tan ^{-1}(C / D)
$$

but this also determines a minimum. The maximum is chosen simply by requiring that $E$ be positive. 
Test 2 is applied by determining whether (11) is constant (within a tolerance TOL) over time. (It should be constant because $L^{i}$ is zero.) Because this test is time consuming, it is done at rare intervals.

\section{BAKINT}

This routine calculates $u_{i}^{*}$ according to (19), (in a similar fashion to that of calculating $u_{i}$ in FORINT) and integrates (27), (28), and (32) with (33), (34), and (38) as boundary conditions. It prints out its results.

\section{DKCALC}

This integrates (31) and (32) with (36) and (37) as boundary conditions, and prints values of $v_{x k}^{i}, v_{k k}^{i}$. At $t=0$, it calculates ok according to (58).

\section{START}

This short routine accepts input information. The input must include the maximum number of iterations, the number of time steps, the tolerances ETA, ETAl, ETA2, CK, and TOL, the initial value of $\bar{k}$, and the initial nominal control history.

\section{BETA}

The optimal perturbation feedback law for small deviations from an optimal trajectory is given by $(22)$, which, in the present problem, may be approximated by,

$$
\delta u_{i}=-H_{u u}^{i} f_{u}^{-1}\left[v_{x x}^{i+1} \delta_{x_{i}}+v_{x k}^{i+1} \delta k\right]
$$

From (58), and since $v_{k}^{i}=\theta^{T}=0$ on an optimal trajectory,

$$
\delta \mathrm{k}=-\mathrm{V}_{\mathrm{kk}}^{\mathrm{i}+1^{-1}} \mathrm{v}_{\mathrm{kx}}^{\mathrm{i}+1} \delta_{\mathrm{x}_{\mathrm{i}+1}}
$$

$t_{\text {or from }}(68), \delta \mathrm{v}_{i}-\delta \mathrm{v}_{0} \approx 0$ 
To first-order in $\Delta t$ (in a problem which originates from a continuous problem), this may be written

$$
\delta_{k}=-v_{k k}^{i+1} v_{k x}^{-1} \delta_{x_{i}}
$$

See section IV. 1.

Thus,

$$
\delta_{u_{i}}=-H_{u u}^{i^{-1}} f_{u}^{i}\left[v_{x x}^{i+1}-v_{x k}^{i+1} v_{k k}^{i+1} v_{k x}^{-1}\right] \delta_{x_{i}}
$$

The coefficient of $\delta x_{i}$ is calculated in BETA, and printed as $\beta_{1}$, $\beta_{2}, \beta_{3}$. 


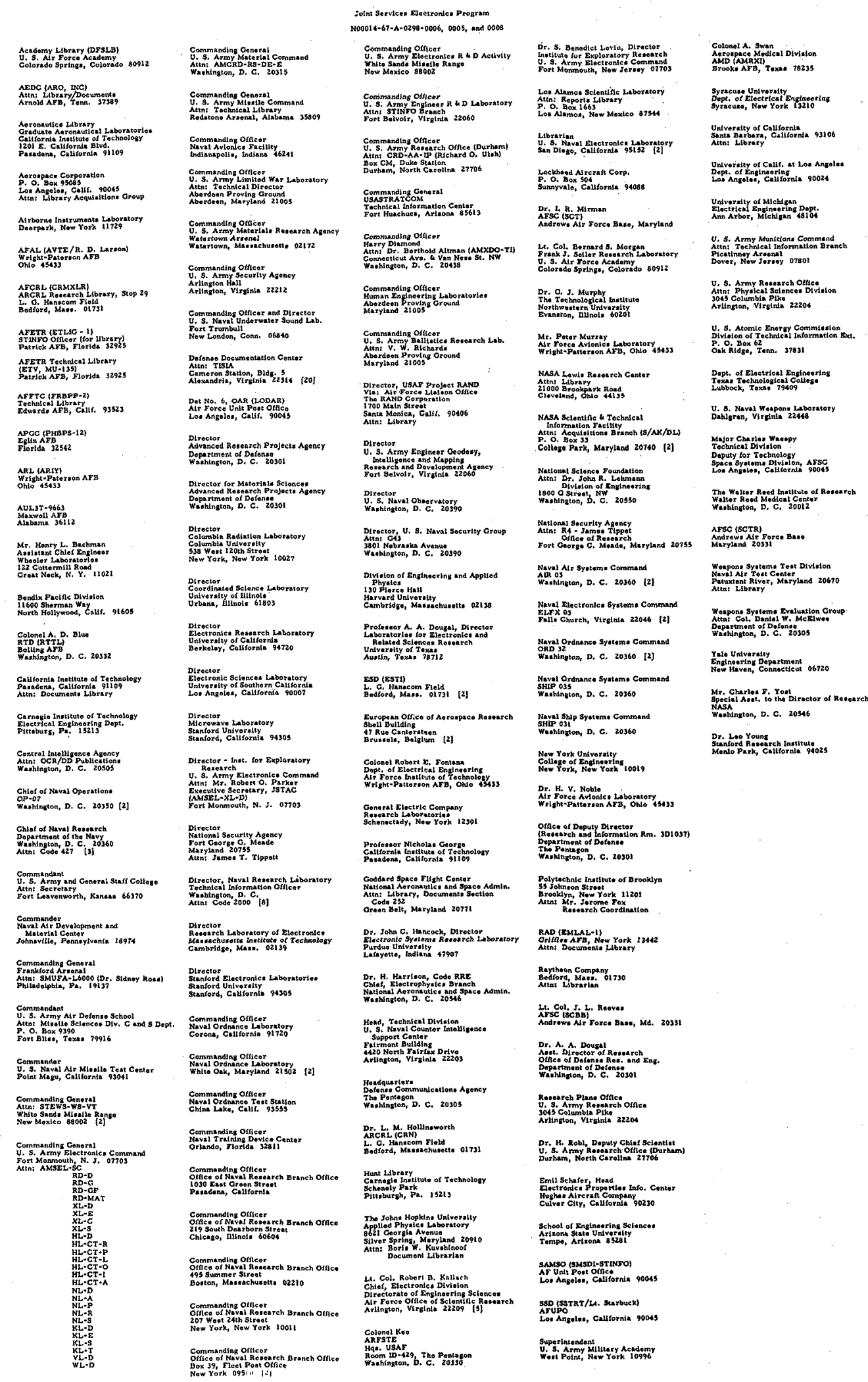


Security classification of title, body of abstract and indexing annotation nust be entered when the overall report is classified) ORIGINATING ACTIVITY (Corporate author)

Division of Engineering and Applied Physics

Harvard University 2a. REPORT SECURITY CLASSIFICATION

Cambridge, Massachusetts

3. REPORT TITLE

A DISCRETE-TIME DIFFERENTIAL DYNAMIC PROGRAMMING ALGORITHM WITH APPLICATION TO OPTIMAL ORBIT TRANSFER

4. PESCRIPTIVE NOTES (TYPe of report and, inclusive dates)

Interim technical report

5. AU THOR(S) (First name, middle initial, last name)

Stanley B. Gershwin and David H. Jacobson

\begin{tabular}{|c|c|c|}
\hline $\begin{array}{l}\text { 6. REPORT DATE } \\
\text { August } 1968\end{array}$ & $\begin{array}{l}\text { 79. TOTAL NO. OF PAGES } \\
60\end{array}$ & $\begin{array}{c}\text { 7b. NO. OF REFS } \\
14 \\
\end{array}$ \\
\hline $\begin{array}{l}\text { Ba.ONTRACTOR GRANTNOO -0006 and NASA } \\
\text { NOO014-67-A-0298-0006 22-007-068 } \\
\text { b. PROJECTNO. Grant NGR 22-007 }\end{array}$ & \multicolumn{2}{|c|}{$\begin{array}{l}\text { 9. ORIGINATOR'S REPORT NUMBER(S) } \\
\text { Technical Report No. } 566\end{array}$} \\
\hline c. & \multicolumn{2}{|c|}{$\begin{array}{l}\text { 9b. OTHER REPORT NO(S) (Any other numbers that may be as signed } \\
\text { this report) }\end{array}$} \\
\hline \multicolumn{3}{|c|}{$\begin{array}{l}\text { 10. DISTRIBUTION STATEMENT } \\
\text { This document has been approved for public } \\
\text { release and sale; its distribution is unlimited. }\end{array}$} \\
\hline 11. SUPPLEMENTARY NOTES & $\begin{array}{r}\text { 12. SPONSORING MILITARY } \\
\text { Office of } N=\end{array}$ & Research \\
\hline
\end{tabular}

Recently, the notion of Differential Dynamic Programming has been used to obtain new second-order algorithms for solving non-linear optimal control problems. (Unlike conventional Dynamic Programming, the Principle of Optimality is applied in the neighborhood of a nominal, non-optimal, trajectory.) A novel feature of these algorithms is that they permit strong variations in the system trajectory.

In this paper, Differential Dynamic Programming is used to develop a second-order algorithm for solving discrete-time dynamic optimization problems with terminal constraints. This algorithm also utilizes strong variations and, as a result, has certain advantages over existing discrete-time methods.

A non-linear computed example is presented, and comparisons are made with the results of other researchers who have solved this problem.

The experience gained during the computation has suggested some extensions to an earlier, previously published Differential Dynamic Programming algorithm for continuous time problems. These extensions, and their implications are discussed. 
Unclassified

Security Classification

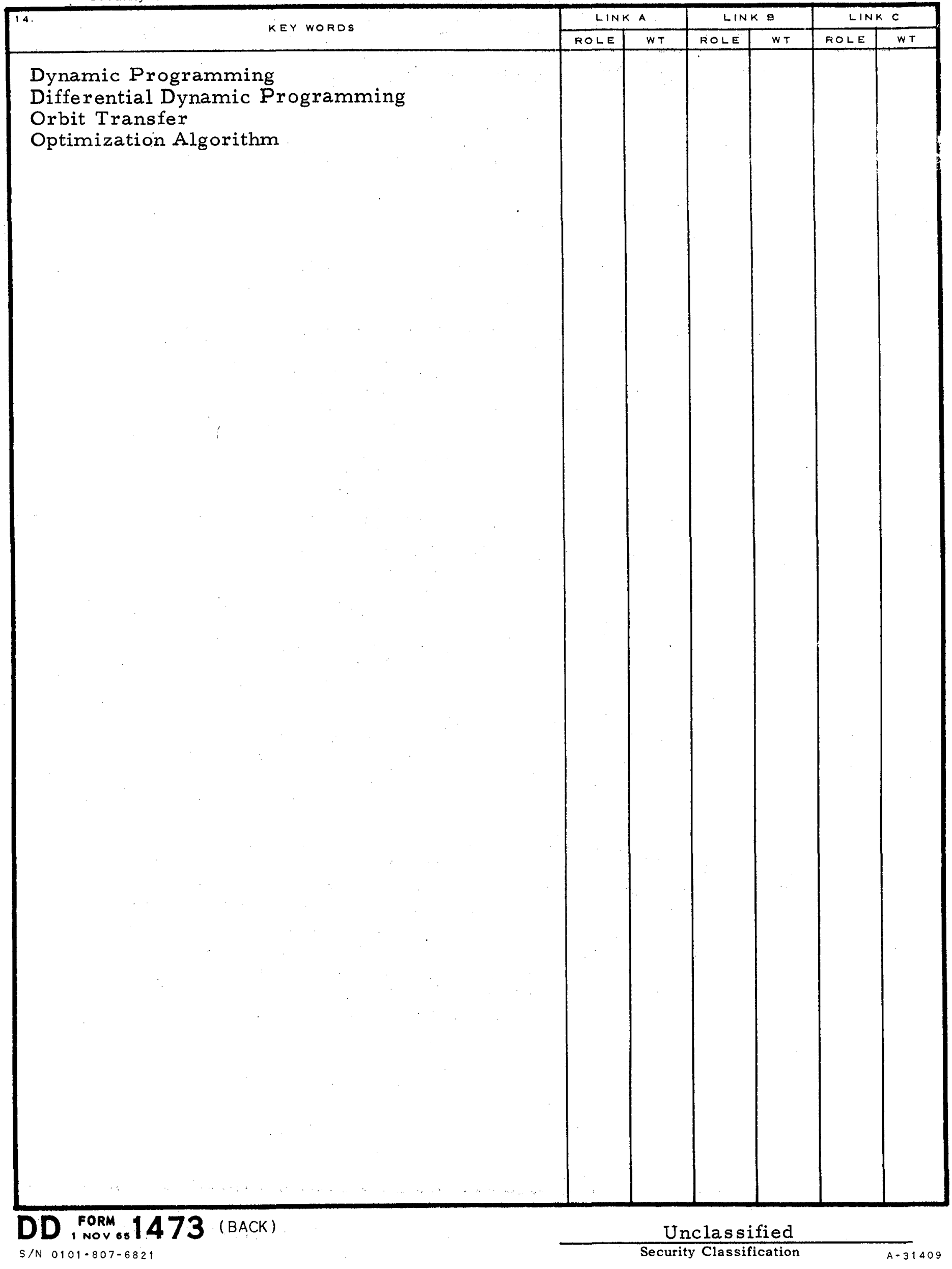

